\title{
Abelian decomposition and glueball-quarkonium mixing in QCD
}

\author{
Pengming Zhang, ${ }^{1}$ Li-Ping Zou, ${ }^{1, *}$ and Y. M. Cho ${ }^{1,2,3, \dagger}$ \\ ${ }^{1}$ Institute of Modern Physics, Chinese Academy of Science, Lanzhou 730000, China \\ ${ }^{2}$ Center for Quantum Spacetime, Sogang University, Seoul 04107, Korea \\ ${ }^{3}$ School of Physics and Astronomy, Seoul National University, Seoul 08826, Korea
}

(Received 27 July 2018; published 26 November 2018)

\begin{abstract}
The Abelian decomposition of QCD which decomposes the gluons to the color neutral binding gluons (the neurons) and the colored valence gluons (the chromons) gauge independently naturally generalizes the quark model to the quark and chromon model which could play the central role in hadron spectroscopy. We discuss the color reflection symmetry, the fundamental symmetry of the quark and chromon model, and explain how it describes the glueballs and the glueball-quarkonium mixing in QCD. We present the numerical analysis of glueball-quarkonium mixing in $0^{++}, 2^{++}$, and $0^{-+}$sectors below $2 \mathrm{GeV}$ and show that in the $0^{++}$sector $f_{0}(500)$ and $f_{0}(1500)$, in the $2^{++}$sector $f_{2}(1950)$, and in the $0^{-+}$sector $\eta(1405)$ and $\eta(1475)$ could be identified as the predominant glueball states. We discuss the physical implications of our result.
\end{abstract}

DOI: 10.1103/PhysRevD.98.096015

\section{INTRODUCTION}

An important issue in hadron spectroscopy is the identification of the glueballs. The general wisdom is that QCD must have glueballs made of gluons [1-3], and several models of glueballs have been proposed [4-9]. Moreover, lattice QCD was able to construct the low-lying glueballs based on the first principles of QCD dynamics [10,11], and the Particle Data Group (PDG) has accumulated a large number of hadronic states that do not seem to fit the simple quark model as the glueball candidates [12].

In spite of the huge efforts to identify the glueballs experimentally, so far the search for the glueballs has not been so successful [13-17]. There are two reasons for this. First, theoretically, there has been no consensus on how to construct the glueballs. This has made it difficult to predict what kind of glueballs we could expect. To see this, consider the two leading models of glueballs - the bag model and the constituent gluon model.

The bag model identifies the glueballs as the gaugeinvariant combinations of the gluon fields confined in a bag $[4,5,18]$. In this model, the confinement is imposed by the boundary condition of the bag, where the interaction among the confined gluons is described by the perturbative

\footnotetext{
* Corresponding author. zoulp@impcas.ac.cn

ymcho0416@gmail.com

Published by the American Physical Society under the terms of the Creative Commons Attribution 4.0 International license. Further distribution of this work must maintain attribution to the author(s) and the published article's title, journal citation, and DOI. Funded by SCOAP ${ }^{3}$.
}

gluon exchange. On the other hand, in the constituent gluon model the glueballs are identified as the color singlet bound states of the color octet "constituent gluons," where the confinement is enforced by the confining potential $[7,8]$.

Intuitively, these models look reasonable and attractive, although they have their own advantages and disadvantages. They were able to show the existence of glueballs. However, they have not been so successful to pinpoint exactly what are the glueball states and tell us how can we verify them.

The other reason is that it is not clear how to identify the glueballs experimentally. This is partly because they could mix with quarkoniums, so that we must take care of the possible mixing to identify the glueballs experimentally [13-17]. This is why we have very few candidates for the glueballs so far, compared to the huge hadron spectrum made of quarks listed in PDG.

This makes the search for the glueballs an urgent issue in high-energy physics, and we have detectors (e.g., GlueX at Jefferson Lab and PANDA at FAIR) specifically designed to search for the glueballs $[19,20]$. To have a successful identification of glueballs, however, we must have a better picture of the glueball.

The Abelian decomposition of QCD allows us to do that [21-24]. It decomposes the QCD gauge potential to the Abelian restricted potential which has the full color gauge degrees of freedom and the gauge covariant valence potential which describes the colored gluons (the chromons) in a gauge-independent way. Moreover, it decomposes the restricted potential further to the nontopological Maxwell part which describes the color neutral binding gluons (the neurons) and the topological Dirac part which describes the non-Abelian monopole. 
This tells that there are two types of gluons which play different roles. The neurons play the role of the binding gluons which bind the colored source, while the chromons play the role of the colored source of QCD. So we can view QCD as the restricted QCD (RCD) made of restricted potential which has the chromons as the colored source.

We emphasize that this is against the common wisdom that all gluons (because of the gauge symmetry) are equal, carrying the same color charge. The Abelian decomposition tells that this is not true, and tells us how to separate the colored chromons from the color neutral neurons unambiguously.

Moreover, the Abelian decomposition allows us to study the role of the monopole, and prove that it is the monopole which is responsible for the confinement in lattice QCD [25-28]. As importantly, it allows us to calculate the QCD effective action and demonstrate the monopole condensation gauge independently [29-31].

However, what is most important for our purpose is that it allows us to have a clear picture of glueballs with which we can identify them. This is because the chromons play the role of the constituent gluons while the neurons bind them, after the confinement sets in. So we can construct the glueballs with a finite number of chromons as the constituent. This generalizes the quark model to the quark and chromon model which provides a new picture of hadrons $[21,22,32]$.

The quark model has been very successful. However, the quark and chromon model has many advantages. It predicts new hadronic states, e.g., the hybrid hadrons made of quarks and chromons. More importantly, it provides a clear picture of glueballs and their mixing with the iso-singlet quarkoniums, and allows us to calculate the gluon content of the mixed states.

Of course, the constituent gluon model can also do that, but this model cannot tell the difference between the binding gluons and the constituent gluons. To understand this, consider the hydrogen atom (or any atom) in QED. Obviously, we have photon as well as electron (and proton and neutron) in it, but only the electron determines the atomic structure of the atom in the periodic table. The photon plays no role in the atomic structure. It is there in the form of the electromagnetic field to provide the binding, not as the constituent which determines the atomic structure of the atom. So we need not know how many of them are in the atom to determine it's place in the periodic table.

Exactly the same way the proton has quarks and gluons, but only the three quarks become the constituent. The gluons inside the proton do not play any role in the baryonic structure of the proton which determine the place of proton in the hadron spectroscopy. This means that they must be the "binding" gluons, not the "constituent" gluons, which (just like the photons in the hydrogen atom) provide only the binding of the quarks in the proton. If so, what are the constituent gluons, and how can we distinguish them from the binding gluons? Obviously, the constituent gluon model does not provide the answer.

The Abelian decomposition naturally resolves this difficulty. It tells that there are indeed two types of gluons, the neurons and the chromons, and in general only the chromons could be treated as the constituent gluons [21,22]. This is because the neurons (like the photons) provide the binding force for colored objects, but the chromons (just like the quarks) become the colored source which make bound states in QCD. So (with few exceptions) only the chromons could be qualified to be the constituent of hadrons.

In this picture, the proton has no constituent chromons. However, we emphasize that this does not mean proton does not contain the chromons at all. Clearly, the three valence quarks which make up the proton can exchange chromons among themselves. Moreover, proton could have an infinite number of "the sea chromons", just as they have the sea quarks. However, obviously, these chromons do not play the role of the constituent.

In the quark and chromon model, one could (in principle) construct an infinite number of glueballs with chromons. So one might ask why experimentally we have not so many candidates of them. One could think of two reasons why this is so. First, the glueballs made of chromons have an intrinsic instability [30,31]. So they have broad widths, broader than the normal hadronic decay width. This means that they have a relatively short life-time. So only the lowlying glueballs could actually be observed experimentally. This is because the chromons, unlike the quarks, tend to annihilate each other in the chromo-electric background. This must be contrasted with quarks, which remain stable inside the hadrons.

This is closely related to the asymptotic freedom (antiscreening) of gluons. It is well known that in QED the strong electric background tends to generate the pair creation of electrons, which makes the charge screening [33-35]. However, in QCD, gluons and quarks play opposite roles in the asymptotic freedom. The quarks enhance the screening while the gluons diminish it to generate the antiscreening $[36,37]$. In fact, in the presence of a chromo-electric background, the chromon loop generates a negative imaginary part but the quark loop generates a positive imaginary part in the QCD effective action. This tells that the chromo-electric field tends to generate the pair annihilation of the chromons [30,31,38-40].

Second, in our model, the glueballs inevitably mix with quarkoniums, so that in general they do not appear as mass eigenstates. So, to identify the glueballs, we have to consider the possible mixing with the quarkoniums. This makes the experimental identification of glueballs a nontrivial matter. This is another reason why the experimental identification of the glueballs so far has not been so successful.

Of course, in rare cases we could have the pure glueballs called the oddballs [7,32]. This is because some of the 
chromoballs have the quantum number $J^{P C}$ which cannot be made possible with $q \bar{q}$. In this case, there is no $q \bar{q}$ which could mix with the oddballs, so that they may exist as pure chromoballs. This makes the identification of the oddballs an important issue in QCD.

In a recent paper, we have discussed the general framework of hadron spectroscopy based on the quark and chromon model, and showed how the model can explain the glueball-quarkonium mixing and allow us to identify the glueballs [32]. The present paper is the sequel of this work in which we extend the preceding work and discuss the numerical analysis of the glueball-quarkonium mixing in more detail to help identify the glueballs without ambiguity.

Our analysis makes it clear that the chromoballs play the central role in the meson spectroscopy, although in general they do not appear as mass eigenstates. In particular, our analysis tells that the chromoballquarkonium mixing makes a deep influence on the $q \bar{q}$ octet-singlet mixing. In fact, in the quark and chromon model the $q \bar{q}$ octet-singlet mixing cannot be discussed without the chromoball-quarkonium mixing, because the chromoball-quarkonium mixing inevitably induces the octet-singlet mixing.

The paper is organized as follows. In Sec. II, we review the Abelian decomposition which decomposes the gluons to the color neutral neurons and the colored chromons to justify the quark and chromon model. In Sec. III, we discuss the color reflection symmetry which replaces the non-Abelian gauge symmetry and becomes the fundamental symmetry of the quark and chromon model. In Sec. IV, we explain how the chromoballs, the bound states of chromons, can be understood as the glueballs in the quark and chromon model. In Sec. V, we discuss the glueballquarkonium mixing mechanism. In Sec. VI, we present the numerical analysis of the low-lying glueball-quarkonium mixing in $0^{++}, 2^{++}$, and $0^{-+}$sectors below $2 \mathrm{GeV}$, and show that $f_{0}(1500), f_{2}(1950), \eta(1405)$, and $\eta(1475)$ become the strong candidates of glueballs. Finally, in the last section, we discuss the physical implications of our analysis.

\section{ABELIAN DECOMPOSITION OF GLUONS: NEURONS AND CHROMONS}

Before we discuss the Abelian decomposition we have to know why we need it. Consider the proton. The quark model tells that it is made of three quarks, but obviously we need the gluon to bind them. On the other hand, the quark model tells that there is no "valence" gluon inside the proton which can be a constituent of the proton. If so, what is the "binding" gluon inside the proton, and how do we distinguish it from the valence gluon?

Another motivation is the Abelian dominance, which asserts that the Abelian part of QCD is responsible for the color confinement $[41,42]$. This must be true, because the
non-Abelian (off-diagonal) part describes the colored gluons which are destined to be confined. Since the confined prisoner cannot be the confining agent (the jailer), only the Abelian part can play the role of the confiner. However, what is the Abelian part, and how do we separate it?

The Abelian decomposition decomposes the QCD gauge potential to the restricted (Abelian) part and the valence (colored) part gauge independently. Consider the SU(2) QCD first, and let $\left(\hat{n}_{1}, \hat{n}_{2}, \hat{n}_{3}=\hat{n}\right)$ be an arbitrary local orthonormal basis. To make the Abelian decomposition, we choose any direction, e.g., $\hat{n}$, to be the Abelian direction and impose the isometry to project out the restricted potential $\hat{A}_{\mu}[21-23]$

$$
\begin{aligned}
D_{\mu} \hat{n} & =\left(\partial_{\mu}+g \vec{A}_{\mu} \times\right) \hat{n}=0, \\
\vec{A}_{\mu} \rightarrow \hat{A}_{\mu} & =A_{\mu} \hat{n}-\frac{1}{g} \hat{n} \times \partial_{\mu} \hat{n}=\mathcal{A}_{\mu}+\mathcal{C}_{\mu}, \\
\mathcal{A}_{\mu} & =A_{\mu} \hat{n}, \quad \mathcal{C}_{\mu}=-\frac{1}{g} \hat{n} \times \partial_{\mu} \hat{n}, \quad A_{\mu}=\hat{n} \cdot \vec{A}_{\mu} .
\end{aligned}
$$

The Abelian projection has the followings features. First, $\hat{A}_{\mu}$ is precisely the potential which leaves the Abelian direction invariant under the parallel transport. Second, it is made of two parts- the nontopological (Maxwellian) $\mathcal{A}_{\mu}$ which describes the color neutral gluon (the neuron) and the topological (Diracian) $\mathcal{C}_{\mu}$ which describes the nonAbelian monopole [43]. Third, the decomposition is gauge independent. We can rotate $\hat{n}$ to any direction and still get exactly the same decomposition.

With this, we have

$$
\begin{aligned}
\hat{F}_{\mu \nu} & =\left(F_{\mu \nu}+H_{\mu \nu}\right) \hat{n}, \\
F_{\mu \nu} & =\partial_{\mu} A_{\nu}-\partial_{\nu} A_{\mu}, \\
H_{\mu \nu} & =-\frac{1}{g} \hat{n} \cdot\left(\partial_{\mu} \hat{n} \times \partial_{\nu} \hat{n}\right) .
\end{aligned}
$$

This tells the followings. First, $\hat{F}_{\mu \nu}$ has only the Abelian component. Second, $\hat{F}_{\mu \nu}$ has a dual structure, made of nontopological $F_{\mu \nu}$ and topological $H_{\mu \nu}$.

With (1), we can recover the full QCD potential adding the non-Abelian (colored) part $\vec{X}_{\mu}$ which describes the colored gluons (the chromons) [21,22]

$$
\vec{A}_{\mu}=\hat{A}_{\mu}+\vec{X}_{\mu}, \quad \hat{n} \cdot \vec{X}_{\mu}=0 .
$$

Under the infinitesimal gauge transformation

$$
\delta \vec{A}_{\mu}=\frac{1}{g} D_{\mu} \vec{\alpha}, \quad \delta \hat{n}_{i}=-\vec{\alpha} \times \hat{n}_{i}
$$


we have

$$
\delta \hat{A}_{\mu}=\frac{1}{g} \hat{D}_{\mu} \vec{\alpha}, \quad \delta \vec{X}_{\mu}=-\vec{\alpha} \times \vec{X}_{\mu},
$$

where $\hat{D}_{\mu}=\partial_{\mu}+g \hat{A}_{\mu} \times$. This tells that $\hat{A}_{\mu}$ has the full $\mathrm{SU}(2)$ gauge degrees of freedom, even though it is restricted. Moreover, $\vec{X}_{\mu}$ becomes gauge covariant.

Notice that, although the neuron is given by the Abelian component of $\vec{A}_{\mu}$, the chromon is not given by the nonAbelian component of $\vec{A}_{\mu}$. This is because the Abelian decomposition decomposes $\vec{A}_{\mu}$ to the neuron, chromon, and the topological monopole. So the topological part plays an essential role in the Abelian decomposition.

With the restricted potential we can construct the restricted QCD (RCD) which has the full non-Abelian gauge symmetry but is simpler than the QCD

$$
\begin{aligned}
\mathcal{L}_{\mathrm{RCD}}= & -\frac{1}{4} \hat{F}_{\mu \nu}^{2} \\
= & -\frac{1}{4} F_{\mu \nu}^{2}+\frac{1}{2 g} F_{\mu \nu} \hat{n} \cdot\left(\partial_{\mu} \hat{n} \times \partial_{\nu} \hat{n}\right) \\
& -\frac{1}{4 g^{2}}\left(\partial_{\mu} \hat{n} \times \partial_{\nu} \hat{n}\right)^{2},
\end{aligned}
$$

which describes the Abelian subdynamics of QCD. Since RCD contains the non-Abelian monopole degrees explicitly, it provides an ideal platform for us to study the monopole dynamics gauge independently.

From (3) we have

$$
\vec{F}_{\mu \nu}=\hat{F}_{\mu \nu}+\hat{D}_{\mu} \vec{X}_{\nu}-\hat{D}_{\nu} \vec{X}_{\mu}+g \vec{X}_{\mu} \times \vec{X}_{\nu}
$$

With this we can express QCD by

$$
\begin{aligned}
\mathcal{L}_{\mathrm{QCD}}= & -\frac{1}{4} \vec{F}_{\mu \nu}^{2} \\
= & -\frac{1}{4} \hat{F}_{\mu \nu}^{2}-\frac{1}{4}\left(\hat{D}_{\mu} \vec{X}_{\nu}-\hat{D}_{\nu} \vec{X}_{\mu}\right)^{2} \\
& -\frac{g}{2} \hat{F}_{\mu \nu} \cdot\left(\vec{X}_{\mu} \times \vec{X}_{\nu}\right)-\frac{g^{2}}{4}\left(\vec{X}_{\mu} \times \vec{X}_{\nu}\right)^{2} .
\end{aligned}
$$

This is the extended SU(2) QCD (ECD) which confirms that QCD can be viewed as RCD made of the binding gluons, which has the chromons as its source $[21,22]$.

The Abelian decomposition is more complicated but straightforward. Since SU(3) has rank two, it has two Abelian directions. Let $\hat{n}_{i}(i=1,2, \ldots, 8)$ be an arbitrary local orthonormal SU(3) basis, and choose $\hat{n}_{3}=\hat{n}$ and $\hat{n}_{8}=\hat{n}^{\prime}$ to be the Abelian directions. Make the Abelian projection by

$$
D_{\mu} \hat{n}=0
$$

This automatically guarantees [43]

$$
D_{\mu} \hat{n}^{\prime}=0, \quad \hat{n}^{\prime}=\frac{1}{\sqrt{3}} \hat{n} * \hat{n} .
$$

where $*$ denotes the $d$-product. This is because $\mathrm{SU}(3)$ has two vector products, the antisymmetric $f$-product and the symmetric $d$-product.

Solving (9), we have the Abelian projection which projects out the binding potential,

$$
\begin{aligned}
\vec{A}_{\mu} \rightarrow \hat{A}_{\mu} & =A_{\mu} \hat{n}+A_{\mu}^{\prime} \hat{n}^{\prime}-\frac{1}{g} \hat{n} \times \partial_{\mu} \hat{n}-\frac{1}{g} \hat{n}^{\prime} \times \partial_{\mu} \hat{n}^{\prime} \\
& =\sum_{p} \frac{2}{3} \hat{A}_{\mu}^{p}, \quad(p=1,2,3), \\
\hat{A}_{\mu}^{p} & =A_{\mu}^{p} \hat{n}^{p}-\frac{1}{g} \hat{n}^{p} \times \partial_{\mu} \hat{n}^{p}=\mathcal{A}_{\mu}^{p}+\mathcal{C}_{\mu}^{p}, \\
\mathcal{A}_{\mu}^{p} & =A_{\mu}^{p} \hat{n}^{p}, \quad \mathcal{C}_{\mu}^{p}=-\frac{1}{g} \hat{n}^{p} \times \partial_{\mu} \hat{n}^{p}, \\
A_{\mu}^{1} & =A_{\mu}, \quad A_{\mu}^{2}=-\frac{1}{2} A_{\mu}+\frac{\sqrt{3}}{2} A_{\mu}^{\prime}, \\
A_{\mu}^{3} & =-\frac{1}{2} A_{\mu}-\frac{\sqrt{3}}{2} A_{\mu}^{\prime}, \quad \hat{n}^{1}=\hat{n}, \\
\hat{n}^{2} & =-\frac{1}{2} \hat{n}+\frac{\sqrt{3}}{2} \hat{n}^{\prime}, \quad \hat{n}^{3}=-\frac{1}{2} \hat{n}-\frac{\sqrt{3}}{2} \hat{n}^{\prime},
\end{aligned}
$$

where the sum is the sum of the Abelian directions of three $\mathrm{SU}(2)$ subgroups made of $\left(\hat{n}_{1}, \hat{n}_{2}, \hat{n}^{1}\right),\left(\hat{n}_{6}, \hat{n}_{7}, \hat{n}^{2}\right)$, $\left(\hat{n}_{4},-\hat{n}_{5}, \hat{n}^{3}\right)$. Notice that the three $\hat{A}_{\mu}^{p}$ are not mutually independent.

Under the infinitesimal gauge transformation

$$
\delta \vec{A}_{\mu}=\frac{1}{g} D_{\mu} \vec{\alpha}, \quad \delta \hat{n}=-\vec{\alpha} \times \hat{n}, \quad \delta \hat{n}^{\prime}=-\vec{\alpha} \times \hat{n}^{\prime},
$$

we have [21-23]

$$
\delta \hat{A}_{\mu}=\frac{1}{g} \hat{D}_{\mu} \vec{\alpha}
$$

This confirms that $\hat{A}_{\mu}$ has the full SU(3) gauge degrees of freedom.

From this we have the restricted field strength made of the binding potential

$$
\begin{aligned}
\hat{F}_{\mu \nu} & =\frac{2}{3} \sum_{p} \hat{F}_{\mu \nu}^{p}=\frac{2}{3} \sum_{p}\left(F_{\mu \nu}^{p}+H_{\mu \nu}^{p}\right)^{2}, \\
F_{\mu \nu}^{p} & =\partial_{\mu} A_{\nu}^{p}-\partial_{\nu} A_{\mu}^{p}, \\
H_{\mu \nu}^{p} & =-\frac{1}{g} \hat{n}^{p} \cdot\left(\partial_{\mu} \hat{n}^{p} \times \partial_{\nu} \hat{n}^{p}\right),
\end{aligned}
$$


and obtain the restricted QCD (RCD) which has the full $\mathrm{SU}(3)$ gauge symmetry [21-23]

$$
\mathcal{L}_{\mathrm{RCD}}=-\frac{1}{4} \hat{F}_{\mu \nu}^{2}=-\frac{1}{6} \sum_{p}\left(F_{\mu \nu}^{p}+H_{\mu \nu}^{p}\right)^{2} .
$$

Just like the SU(2) RCD it has a dual structure, made of $F_{\mu \nu}^{p}$ and $H_{\mu \nu}^{p}$.

Adding the valence part $\vec{X}_{\mu}$ which describes the chromons to the binding potential we have the Abelian decomposition of the SU(3) gauge potential [44]

$\vec{A}_{\mu}=\hat{A}_{\mu}+\vec{X}_{\mu}=\sum_{p}\left(\frac{2}{3} \hat{A}_{\mu}^{p}+\vec{W}_{\mu}^{p}\right), \quad \vec{X}_{\mu}=\sum_{p} \vec{W}_{\mu}^{p}$,

$\vec{W}_{\mu}^{1}=X_{\mu}^{1} \hat{n}_{1}+X_{\mu}^{2} \hat{n}_{2}, \quad \vec{W}_{\mu}^{2}=X_{\mu}^{6} \hat{n}_{6}+X_{\mu}^{7} \hat{n}_{7}$,

$\vec{W}_{\mu}^{3}=X_{\mu}^{4} \hat{n}_{4}+X_{\mu}^{5} \hat{n}_{5}$.

Again, under the gauge transformation we have [21-23]

$$
\delta \hat{A}_{\mu}=\frac{1}{g} \hat{D}_{\mu} \vec{\alpha}, \quad \delta \vec{X}_{\mu}=-\vec{\alpha} \times \vec{X}_{\mu}
$$

This confirms that $\vec{X}_{\mu}$ becomes gauge covariant. Moreover, this tells that the chromons $\vec{X}_{\mu}$ can be decomposed to the three $\mathrm{SU}(2)$ chromons $\vec{W}_{\mu}^{p}$. However, unlike $\hat{A}_{\mu}^{p}$, they are mutually independent. So we have two neurons and six chromons in SU(3) QCD. The Abelian decomposition has also been known as the Cho decomposition, ChoDuan-Ge decomposition, or Cho-Faddeev-Niemi decomposition [45-49].

From (16), we have

$$
\begin{aligned}
\hat{D}_{\mu} \vec{X}_{\nu}= & \sum_{p} \hat{D}_{\mu}^{p} \vec{W}_{\nu}^{p}, \quad \hat{D}_{\mu}^{p}=\partial_{\mu}+g \hat{A}_{\mu}^{p} \times, \\
\vec{X}_{\mu} \times \vec{X}_{\nu}= & \sum_{p, q} \vec{W}_{\mu}^{p} \times \vec{W}_{\nu}^{q}, \\
\vec{F}_{\mu \nu}= & \hat{F}_{\mu \nu}+\hat{D}_{\mu} \vec{X}_{\nu}-\hat{D}_{\nu} \vec{X}_{\mu}+g \vec{X}_{\mu} \times \vec{X}_{\nu} \\
= & \sum_{p}\left[\frac{2}{3} \hat{F}_{\mu \nu}^{p}+\left(\hat{D}_{\mu}^{p} \vec{W}_{\nu}^{p}-\hat{D}_{\mu}^{p} \vec{W}_{\nu}^{p}\right)\right] \\
& +\sum_{p, q} \vec{W}_{\mu}^{p} \times \vec{W}_{\nu}^{q},
\end{aligned}
$$

so that we can express the SU(3) QCD as [29-31]

$$
\begin{aligned}
\mathcal{L}_{\mathrm{ECD}}= & \mathcal{L}_{\mathrm{RCD}}-\frac{1}{4}\left(\hat{D}_{\mu} \vec{X}_{\nu}-\hat{D}_{\nu} \vec{X}_{\mu}\right)^{2}-\frac{g}{2}\left(\hat{D}_{\mu} \vec{X}_{\nu}-\hat{D}_{\nu} \vec{X}_{\mu}\right) \cdot\left(\vec{X}_{\mu} \times \vec{X}_{\nu}\right)-\frac{g}{2} \hat{F}_{\mu \nu} \cdot\left(\vec{X}_{\mu} \times \vec{X}_{\nu}\right)-\frac{g^{2}}{4}\left(\vec{X}_{\mu} \times \vec{X}_{\nu}\right)^{2} \\
= & \sum_{p}\left[-\frac{1}{6}\left(\hat{F}_{\mu \nu}^{p}\right)^{2}-\frac{1}{4}\left(\hat{D}_{\mu}^{p} \vec{W}_{\nu}^{p}-\hat{D}_{\nu}^{p} \vec{W}_{\mu}^{p}\right)^{2}-\frac{g}{2} \hat{F}_{\mu \nu}^{p} \cdot\left(\vec{W}_{\mu}^{p} \times \vec{W}_{\nu}^{p}\right)\right]-\sum_{p, q} \frac{g^{2}}{4}\left(\vec{W}_{\mu}^{p} \times \vec{W}_{\mu}^{q}\right)^{2} \\
& -\sum_{p, q, r} \frac{g}{2}\left(\hat{D}_{\mu}^{p} \vec{W}_{\nu}^{p}-\hat{D}_{\nu}^{p} \vec{W}_{\mu}^{p}\right) \cdot\left(\vec{W}_{\mu}^{q} \times \vec{W}_{\mu}^{r}\right)-\sum_{p \neq q} \frac{g^{2}}{4}\left[\left(\vec{W}_{\mu}^{p} \times \vec{W}_{\nu}^{q}\right) \cdot\left(\vec{W}_{\mu}^{q} \times \vec{W}_{\nu}^{p}\right)+\left(\vec{W}_{\mu}^{p} \times \vec{W}_{\nu}^{p}\right) \cdot\left(\vec{W}_{\mu}^{q} \times \vec{W}_{\nu}^{q}\right)\right] .
\end{aligned}
$$

This is the SU(3) ECD, which is mathematically identical to QCD. Adding an extra term or subtracting any existing term is strictly forbidden.

We can easily add quarks in the Abelian decomposition,

$$
\begin{aligned}
\mathcal{L}_{q} & =\sum_{k} \bar{\Psi}_{k}\left(i \gamma^{\mu} D_{\mu}-m\right) \Psi_{k} \\
& =\sum_{k}\left[\bar{\Psi}_{k}\left(i \gamma^{\mu} \hat{D}_{\mu}-m\right) \Psi_{k}+\frac{g}{2} \vec{X}_{\mu} \cdot \bar{\Psi}_{k}\left(\gamma^{\mu} \vec{t}\right) \Psi_{k}\right] \\
& =\sum_{p, k}\left[\bar{\Psi}_{k}^{p}\left(i \gamma^{\mu} \hat{D}_{\mu}^{p}-m\right) \Psi_{k}^{p}+\frac{g}{2} \vec{W}_{\mu}^{p} \cdot \bar{\Psi}_{k}^{p}\left(\gamma^{\mu} \vec{\tau}^{p}\right) \Psi_{k}^{p}\right] \\
\hat{D}_{\mu} & =\partial_{\mu}+\frac{g}{2 i} \vec{t} \cdot \hat{A}_{\mu}, \quad \hat{D}_{\mu}^{p}=\partial_{\mu}+\frac{g}{2 i} \vec{\tau}^{p} \cdot \hat{A}_{\mu}^{p},
\end{aligned}
$$

where $m$ is the mass, $k$ is the flavor index, $\vec{t}$ is the color generators of the quark triplet corresponding to the chromons $\vec{X}_{\mu}, p$ denotes the color generators of the quarks corresponding to three $\mathrm{SU}(2)$ subgroups of $\mathrm{SU}(3)$, and $\Psi_{k}^{p}$ represents the three $\mathrm{SU}(2)$ quark doublets (i.e., $(r, b),(b, g)$, and $(g, r)$ doublets) of the $(r, b, g)$ quark triplet.

The Abelian decomposition is summarized graphically. This is shown in Fig. 1, where the gluons are decomposed to the restricted potential and the chromon potential in (A), and the restricted potential is decomposed further to the nontopological neuron potential $\mathcal{A}_{\mu}$ and the topological monopole potential $\mathcal{C}_{\mu}$ in (B).

Although the Abelian decomposition does not change QCD, it reveals important hidden structures of QCD. First of all, it tells that there are two types of gluon, the neuron and chromon, which play totally different role. This means that there should be two types of gluon jets, the neuron jet and the chromon jet, which in principle could be tested and confirmed by experiment. Without the Abelian decomposition we could not tell this because all gluons are treated on equal footing.

Second, it allows us to decompose the QCD Feynman diagram in such a way that the conservation of color is 


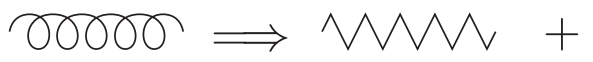

(a)

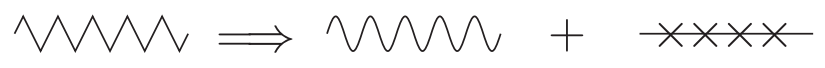

(b)

FIG. 1. The Abelian decomposition of the gluons. The gauge potential is decomposed to the restricted potential (kinked line) and the chromon (straight line) in (A), and the restricted potential is further decomposed to the neuron (wiggly line) and the monopole (spiked line) in (B).

made explicit. This is shown in Fig. 2. In (A), the threepoint gluon vertex is decomposed to two vertices made of one neuron plus two chromons and three chromons. In (B), the four-point gluon vertex is decomposed to three vertices made of one neuron plus three chromons, two neurons plus two chromons, and four chromons. In (C), the quark-gluon vertex is decomposed to the quark-neuron vertex and quark-chromon vertex.

Notice that three-point vertex made of three neurons or two neurons and one chromon, and four-point vertex made of three or four neurons are forbidden by the conservation of color. Moreover, the quark-neuron interaction does not change the quark color, but the quark-chromon interaction changes the quark color.

Another point is that the monopole does not appear in the diagram for the following reasons. First, after the confinement (the monopole condensation) sets in, the monopole

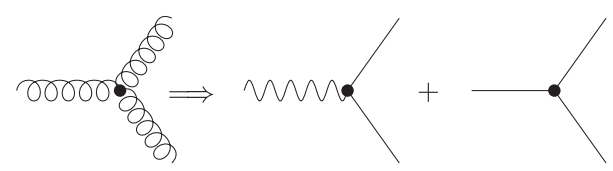

(a)

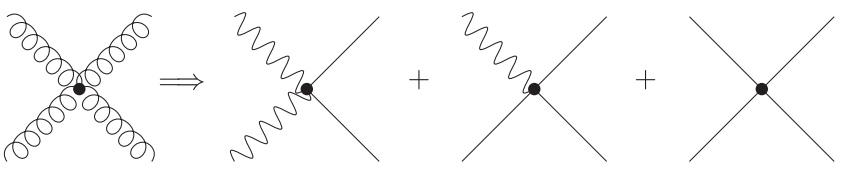

(b)

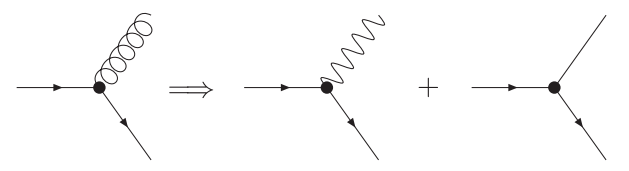

(c)

FIG. 2. The Abelian decomposition of Feynman diagrams in SU(3) QCD. The three-point and four-point gluon vertices are decomposed in (A) and (B), and the quark-gluon vertices are decomposed in $(\mathrm{C})$. Notice that the monopole does not appear in the Feynman diagram, since it does not represent a dynamical degree. part disappears completely. So, in the perturbative regime (inside the hadrons where the asymptotic freedom applies) only the neurons and chromons contribute to the Feynman diagrams. However, the more fundamental reason is that the monopole, as the topological degree of QCD, does not become a dynamical (i.e., propagating) degree. So, it cannot appear in the Feynman diagram [29].

Third, the Abelian decomposition of SU(3) QCD reveals the Weyl symmetry of the SU(3) QCD, and shows that the theory is invariant under the permutation of three $\mathrm{SU}(2)$ subgroups, or equivalently three colors of SU(3). Indeed, (11), (19), and (20) clearly show that they are invariant under the permutation of three SU(2) subgroups. The Weyl group of $\mathrm{SU}(\mathrm{N})$ is the $N$ !-elements permutation group of $N$ colors. In general, the Abelian decomposition allows us to express the SU(N) QCD explicitly in the Weyl symmetric form. This is very important, because this allows us to express the SU(N) QCD effective action in terms of the SU(2) QCD effective action.

In the nonperturbative regime, the Abelian decomposition allows us to demonstrate the monopole dominance, that it is the monopole which confines the color. In fact, implementing the Abelian decomposition on lattice, we can calculate the contribution of the Wilson loop with the full potential, the restricted potential, and the monopole potential separately, and show that the monopole potential produces the confining force [25-28]. The recent lattice result obtained with the Abelian decomposition is copied in Fig. 3, which shows that all three potentials produce exactly the same confining force $[27,28]$. Clearly, this proves that the neuron and chromon do not contribute to the Wilson loop integral.

The lattice result demonstrates the monopole dominance, that the monopole is essential for the confinement. However, it does not show how the monopole confines

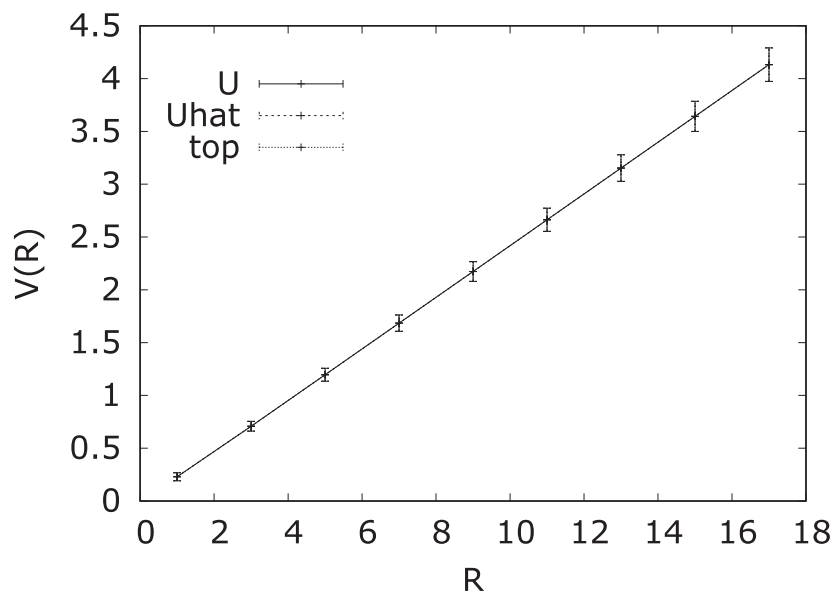

FIG. 3. The lattice QCD calculation which establishes the monopole dominance in Wilson loop. Here, the solid, dotted, and dashed lines are obtained with the full potential, the restricted potential, and the monopole potential, respectively. 


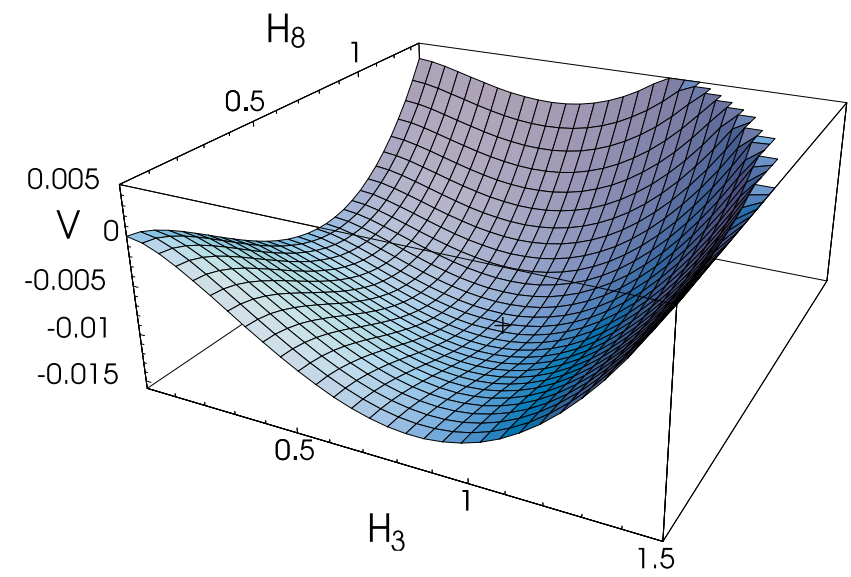

FIG. 4. The one-loop effective potential of SU(3) QCD which demonstrates the monopole condensation. The potential is obtained by integrating out the chromons in the presence of a constant monopole background.

the color. To show this, we have to calculate the effective action of QCD. The Abelian decomposition and the resulting ECD provides us an ideal platform for us to calculate the QCD effective action gauge independently $[30,31]$.

This is because, field theoretically, the Abelian decomposition puts QCD in the background field formalism $[29,50,51]$. So we can treat the restricted potential and the valence potential as the slow varying classical field and the fluctuating quantum field, and calculate the QCD effective action in the presence of the monopole background imposing the gauge invariance.

This allows us to show that the true QCD vacuum is given by the monopole condensation, more precisely the monopole-antimonopole pair condensation [30,31]. The SU(3) QCD effective potential is shown in Fig. 4. This strongly implies that it is the monopole condensation which generates the mass gap and the confinement in QCD.

The most important point of the Abelian decomposition for our purpose in this paper, however, is that it generalizes the quark model to the quark and chromon model [32]. This is because the chromons, being colored, are naturally qualified to become the constituent of hadrons. In contrast, the neurons, being neutral, play the role of photons which provides the binding in QED.

This leads us to the quark and chromon model where the colored quarks and chromons become the constituent of hadrons. This gives us a new picture of hadron spectroscopy. Moreover, this provides us a clear picture of the glueballs and their mixing with quarkoniums and helps us to identify the glueballs experimentally.

To understand how the quark and chromon model works, it is important to understand that the Abelian decomposition reduces the complicated non-Abelian color gauge symmetry to the discrete symmetry made of finite elements called the color reflection symmetry which becomes the fundamental symmetry of the quark and chromon model $[21,22]$. This is very important, because this color reflection symmetry plays the role of the gauge symmetry but is much easier to handle. So we discuss the color reflection symmetry in detail in the next section.

\section{COLOR REFLECTION INVARIANCE AND WEYL SYMMETRY OF ECD}

As we have emphasized, the Abelian decomposition is gauge independent. On the other hand, the selection of the Abelian direction amounts to the gauge fixing which breaks the gauge symmetry. However, this does not break the gauge symmetry completely, because we have a residual discrete symmetry called the color reflection symmetry even after the Abelian decomposition [21,22,30].

The importance of this residual symmetry comes from the following observation. First, this plays the role of the gauge symmetry after the Abelian decomposition. Second, this symmetry is much simpler than the color gauge symmetry. This tells that the Abelian decomposition reduces the complicated non-Abelian gauge symmetry to a simple discrete symmetry which is much easier to handle. So we discuss the color reflection symmetry first.

Consider the SU(2) QCD first and make the color reflection, the $\pi$-rotation of the $\mathrm{SU}(2)$ basis along the $\hat{n}_{2}$-direction which inverts the color direction $\hat{n}$,

$$
\left(\hat{n}_{1}, \hat{n}_{2}, \hat{n}\right) \rightarrow\left(-\hat{n}_{1}, \hat{n}_{2},-\hat{n}\right)
$$

Obviously, this is a gauge transformation which should not change the physics. On the other hand, under the color reflection (21) we have [30]

$$
\begin{aligned}
& \hat{A}_{\mu} \rightarrow \hat{A}_{\mu}^{(c)}=-A_{\mu} \hat{n}-\frac{1}{g} \hat{n} \times \partial_{\mu} \hat{n}, \\
& A_{\mu} \rightarrow A_{\mu}^{(c)}=-\hat{n} \cdot \vec{A}_{\mu}=-A_{\mu} .
\end{aligned}
$$

Moreover, we have

$$
\vec{X}_{\mu} \rightarrow \vec{X}_{\mu}^{(c)}=-\left(X_{\mu}^{1} \hat{n}_{1}-X_{\mu}^{2} \hat{n}_{2}\right)
$$

or, in the complex notation

$$
\begin{aligned}
R_{\mu} & =\frac{1}{\sqrt{2}}\left(X_{\mu}+i X_{\mu}^{2}\right) \\
\rightarrow R_{\mu}^{(c)} & =-\bar{R}_{\mu}=-\frac{1}{\sqrt{2}}\left(X_{\mu}-i X_{\mu}^{2}\right),
\end{aligned}
$$

where $R_{\mu}$ denotes the red chromon.

However, since the isometry condition (1) is insensitive to (21), we have two different Abelian decompositions imposing the same isometry, 


$$
\vec{A}_{\mu}=\hat{A}_{\mu}+\vec{X}_{\mu}, \quad \vec{A}_{\mu}=\hat{A}_{\mu}^{(c)}+\vec{X}_{\mu}^{(c)},
$$

without changing the physics. This is why the color reflection (21) becomes a discrete symmetry of QCD after the Abelian decomposition [21,22].

To understand the meaning of this, notice that the neuron potential $A_{\mu}$ change the signature, while the topological part remains invariant. Moreover the chromon changes to the complex conjugate partner (together with the change of the signature), which changes the chromon to antichromon and flips the sign of the chromon charge.

This is what is expected. In the absence of the topological part, (8) describes QED which is coupled to the massless charged vector field where the neuron plays the role of the photon. And in QED it is well known that the photon has negative charge conjugation quantum number. So it is natural that $A_{\mu}$ in $\mathrm{SU}(2) \mathrm{QCD}$ changes the signature under the color reflection. Similarly we can argue that $A_{\mu}$ changes the signature under the parity [30].

As importantly, (23) tells that the physics should not change when we change the chromon to antichromon, because they are the color reflection partner. This means that they cannot be separately discussed in QCD and should always play exactly the same amount of role. This is the reason why the color should become unphysical and confined, which makes QCD totally different from QCD $[21,22,30]$.

In the fundamental representation, the color reflection (21) is given by the four-element subgroup of $\mathrm{SU}(2)$ made of $[21,22]$

$$
\begin{aligned}
C_{1} & =\left(\begin{array}{ll}
1 & 0 \\
0 & 1
\end{array}\right), & C_{2} & =\left(\begin{array}{cc}
-1 & 0 \\
0 & -1
\end{array}\right), \\
C_{3} & =\left(\begin{array}{cc}
0 & 1 \\
-1 & 0
\end{array}\right), & C_{4} & =\left(\begin{array}{cc}
0 & -1 \\
1 & 0
\end{array}\right) .
\end{aligned}
$$

This can be expressed by

$$
\begin{array}{ll}
C_{k}=D_{a} R_{b}, & (a=1,2 ; b=1,2 ; k=1,2, \ldots, 4), \\
D_{1}=\left(\begin{array}{ll}
1 & 0 \\
0 & 1
\end{array}\right), & D_{2}=\left(\begin{array}{cc}
-1 & 0 \\
0 & -1
\end{array}\right) \\
R_{1}=\left(\begin{array}{ll}
1 & 0 \\
0 & 1
\end{array}\right), & R_{2}=\left(\begin{array}{cc}
0 & 1 \\
-1 & 0
\end{array}\right),
\end{array}
$$

which contains the diagonal subgroup made of $D_{1}$ and $D_{2}$. And this becomes the residual symmetry of the SU(2) quark doublet $(r, b)$ after the Abelian decomposition. Notice that $R_{2}$ plays the role of the generator of the color reflection group.

As for the gluons which form the adjoint representation the color reflection can be simplified further for two reasons. First, the diagonal subgroup has no effect on the adjoint representation. Second, the color reflection changes $\hat{n}$ to $-\hat{n}$ and $\left(\hat{n}_{1}, \hat{n}_{2}\right)$ to $\left(-\hat{n}_{1}, \hat{n}_{2}\right)$. So, the gluon triplet is decomposed to two independent representations. Indeed, for the neuron, we have

$$
R_{2}: A_{\mu} \rightarrow-A_{\mu}
$$

However, for the chromon, we have

$$
R_{2}:\left(\vec{X}_{\mu}, \vec{X}_{\mu}^{(c)}\right) \rightarrow-\left(\vec{X}_{\mu}^{(c)}, \vec{X}_{\mu}\right),
$$

or equivalently

$$
R_{2}:\left(R_{\mu}, \bar{R}_{\mu}\right) \rightarrow-\left(\bar{R}_{\mu}, R_{\mu}\right) .
$$

This confirms that the neuron and chromon transform independently, forming a one-dimensional and twodimensional representation under the color reflection. This drastically simplifies the non-Abelian gauge symmetry.

For SU(3), the fundamental representation of the color reflection group is made up of a 24-element subgroup of $\mathrm{SU}(3)$ given by $[21,22,49]$

$C_{k}=D_{a} R_{b}$,

$(a=1,2,3,4 ; b=1,2, \ldots, 6 ; k=1,2, \ldots, 24)$,

$D_{1}=\left(\begin{array}{ccc}1 & 0 & 0 \\ 0 & 1 & 0 \\ 0 & 0 & 1\end{array}\right), \quad D_{2}=\left(\begin{array}{ccc}-1 & 0 & 0 \\ 0 & -1 & 0 \\ 0 & 0 & 1\end{array}\right)$,

$D_{3}=\left(\begin{array}{ccc}1 & 0 & 0 \\ 0 & -1 & 0 \\ 0 & 0 & -1\end{array}\right), \quad D_{4}=\left(\begin{array}{ccc}-1 & 0 & 0 \\ 0 & 1 & 0 \\ 0 & 0 & -1\end{array}\right)$,

$R_{1}=\left(\begin{array}{lll}1 & 0 & 0 \\ 0 & 1 & 0 \\ 0 & 0 & 1\end{array}\right), \quad R_{2}=\left(\begin{array}{ccc}0 & 1 & 0 \\ -1 & 0 & 0 \\ 0 & 0 & 1\end{array}\right)$,

$R_{3}=\left(\begin{array}{ccc}1 & 0 & 0 \\ 0 & 0 & 1 \\ 0 & -1 & 0\end{array}\right), \quad R_{4}=\left(\begin{array}{ccc}0 & 0 & 1 \\ 0 & -1 & 0 \\ 1 & 0 & 0\end{array}\right)$,

$R_{5}=\left(\begin{array}{lll}0 & 1 & 0 \\ 0 & 0 & 1 \\ 1 & 0 & 0\end{array}\right), \quad R_{6}=\left(\begin{array}{ccc}0 & 0 & 1 \\ -1 & 0 & 0 \\ 0 & -1 & 0\end{array}\right)$,

where the four $D$-matrices form the diagonal subgroup. This describes the residual symmetry of the quark triplet $(r, b, g)$ after the Abelian decomposition. Notice that here $R_{2}$ and $R_{3}$ play the role of the generator. For example, we have $R_{5}=R_{3} \cdot R_{2}, \quad R_{6}=R_{2} \cdot R_{3}$, and $R_{4}=R_{2} \cdot R_{3} \cdot R_{2}$.

For the gluon octet which form the adjoint representation of SU(3) the color reflection can be simplified further. Just as in $\mathrm{SU}(2) \mathrm{QCD}$, the neurons and chromons transform 
separately, among themselves. To see exactly how they transform, notice that the two neurons transform as

$$
\begin{aligned}
R_{2}:\left(\begin{array}{l}
A_{\mu} \\
A_{\mu}^{\prime}
\end{array}\right) \rightarrow\left(\begin{array}{cc}
-1 & 0 \\
0 & 1
\end{array}\right)\left(\begin{array}{l}
A_{\mu} \\
A_{\mu}^{\prime}
\end{array}\right), \\
R_{3}:\left(\begin{array}{l}
A_{\mu} \\
A_{\mu}^{\prime}
\end{array}\right) \rightarrow\left(\begin{array}{cc}
1 / 2 & \sqrt{3} / 2 \\
\sqrt{3} / 2 & -1 / 2
\end{array}\right)\left(\begin{array}{l}
A_{\mu} \\
A_{\mu}^{\prime}
\end{array}\right),
\end{aligned}
$$

On the other hand, according to (11) and (16) the two neurons form a (mutually dependent) triplet $\left(A_{\mu}^{1}, A_{\mu}^{2}, A_{\mu}^{3}\right)$. So in terms of the triplet the color reflection acts as follows,

$$
\begin{aligned}
& R_{2}:\left(A_{\mu}^{1}, A_{\mu}^{2}, A_{\mu}^{3}\right) \rightarrow-\left(A_{\mu}^{1}, A_{\mu}^{3}, A_{\mu}^{2}\right), \\
& R_{3}:\left(A_{\mu}^{1}, A_{\mu}^{2}, A_{\mu}^{3}\right) \rightarrow-\left(A_{\mu}^{3}, A_{\mu}^{2}, A_{\mu}^{1}\right), \\
& R_{4}:\left(A_{\mu}^{1}, A_{\mu}^{2}, A_{\mu}^{3}\right) \rightarrow-\left(A_{\mu}^{2}, A_{\mu}^{1}, A_{\mu}^{3}\right), \\
& R_{5}:\left(A_{\mu}^{1}, A_{\mu}^{2}, A_{\mu}^{3}\right) \rightarrow\left(A_{\mu}^{3}, A_{\mu}^{1}, A_{\mu}^{2}\right), \\
& R_{6}:\left(A_{\mu}^{1}, A_{\mu}^{2}, A_{\mu}^{3}\right) \rightarrow\left(A_{\mu}^{2}, A_{\mu}^{3}, A_{\mu}^{1}\right) .
\end{aligned}
$$

This tells that basically $R_{2}, R_{3}, R_{4}$ describe the permutations of two $\mathrm{SU}(2)$ neurons (up to the signature change), but $R_{5}, R_{6}$ describe the cyclic permutations of three $\mathrm{SU}(2)$ neurons.

For the six chromons which form a sextet $\left(\vec{W}_{\mu}^{1}, \vec{W}_{\mu}^{2}\right.$, $\vec{W}_{\mu}^{3}, \vec{W}_{\mu}^{1(c)}, \vec{W}_{\mu}^{2(c)}, \vec{W}_{\mu}^{3(c)}$ ) we can express them as three (red, blue, and green) colored chromons of the SU(2) subgroups by $\left(R_{\mu}, B_{\mu}, G_{\mu}, \bar{R}_{\mu}, \bar{B}_{\mu}, \bar{G}_{\mu}\right)$. For these the color reflection acts as follows,

$$
\begin{aligned}
R_{2} & :\left(R_{\mu}, B_{\mu}, G_{\mu}, \bar{R}_{\mu}, \bar{B}_{\mu}, \bar{G}_{\mu}\right) \\
& \rightarrow\left(\bar{R}_{\mu}, \bar{G}_{\mu}, \bar{B}_{\mu}, R_{\mu}, G_{\mu}, B_{\mu}\right), \\
R_{3} & :\left(R_{\mu}, B_{\mu}, G_{\mu}, \bar{R}_{\mu}, \bar{B}_{\mu}, \bar{G}_{\mu}\right) \\
& \rightarrow-\left(\bar{G}_{\mu}, \bar{B}_{\mu}, \bar{R}_{\mu}, G_{\mu}, B_{\mu}, R_{\mu}\right), \\
R_{4} & :\left(R_{\mu}, B_{\mu}, G_{\mu}, \bar{R}_{\mu}, \bar{B}_{\mu}, \bar{G}_{\mu}\right) \\
& \rightarrow-\left(\bar{B}_{\mu}, \bar{R}_{\mu}, \bar{G}_{\mu}, B_{\mu}, R_{\mu}, G_{\mu}\right), \\
R_{5} & :\left(R_{\mu}, B_{\mu}, G_{\mu}, \bar{R}_{\mu}, \bar{B}_{\mu}, \bar{G}_{\mu}\right) \\
& \rightarrow-\left(G_{\mu}, R_{\mu}, B_{\mu}, \bar{G}_{\mu}, \bar{R}_{\mu}, \bar{B}_{\mu}\right), \\
R_{6} & :\left(R_{\mu}, B_{\mu}, G_{\mu}, \bar{R}_{\mu}, \bar{B}_{\mu}, \bar{G}_{\mu}\right) \\
& \rightarrow-\left(B_{\mu}, G_{\mu}, R_{\mu}, \bar{B}_{\mu}, \bar{G}_{\mu}, \bar{R}_{\mu}\right) .
\end{aligned}
$$

Here, $R_{2}, R_{3}, R_{4}$ describe the antichromon transformation (complex conjugation) plus permutations of two chromons, but $R_{5}, R_{6}$ describe the cyclic permutations of three chromons (up to the signature change).

The above discussion reveals another important difference between the neuron and chromon. Clearly, (31) tells that the neurons just permute and change the signature of the wave function, but (32) tells that the chromons change to antichromons, under the color reflection. In other words, just like the photon in QED, there are no antineurons in QCD. In contrast, the chromons have the antichromon partners. This is because the neurons are neutral but the chromons are colored, so that the neuron wave functions have real form, while the chromon wave functions have complex expression.

At this point one might wonder if there is any relation between the color reflection group and Weyl group. For $\mathrm{SU}(3)$, the Weyl group is the six-element permutation group of three colors which has a three-dimensional representation given by

$$
\begin{aligned}
W_{1}=\left(\begin{array}{lll}
1 & 0 & 0 \\
0 & 1 & 0 \\
0 & 0 & 1
\end{array}\right), & W_{2}=\left(\begin{array}{lll}
0 & 1 & 0 \\
1 & 0 & 0 \\
0 & 0 & 1
\end{array}\right), \\
W_{3}=\left(\begin{array}{lll}
1 & 0 & 0 \\
0 & 0 & 1 \\
0 & 1 & 0
\end{array}\right), & W_{4}=\left(\begin{array}{lll}
0 & 0 & 1 \\
0 & 1 & 0 \\
1 & 0 & 0
\end{array}\right), \\
W_{5}=\left(\begin{array}{lll}
0 & 1 & 0 \\
0 & 0 & 1 \\
1 & 0 & 0
\end{array}\right), & W_{6}=\left(\begin{array}{lll}
0 & 0 & 1 \\
1 & 0 & 0 \\
0 & 1 & 0
\end{array}\right),
\end{aligned}
$$

which contains the cyclic $Z_{3}$ made of $W_{1}, W_{5}$, and $W_{6}$.

This tells that the two groups are different. They have different origin. The Weyl group comes as the symmetry of the Abelian decomposition, but the color reflection group is the residual symmetry of the Abelian decomposition. Unlike the color reflection group (29), the Weyl group (33) is not a subgroup of SU(3). Moreover, the Weyl group has no complex conjugation operation which transforms the chromons to antichromons. On the other hand, they have a common subgroup $Z_{3}$, the cyclic permutation group of three colors.

Obviously, both the color reflection group and the Weyl group should play a fundamental role in hadron spectroscopy. Only the color reflection-invariant and Weylinvariant combinations of quarks and gluons can become physical in the quark and chromon model [32]. On the other hand, the color reflection group plays a more fundamental role in the sense that it has the complex conjugation operation which transforms the chromons to antichromons.

\section{GLUEBALLS IN QUARK AND CHROMON MODEL: CHROMOBALLS}

So far, we have discussed the theoretical aspects of QCD which are exact. From now on we discuss their applications which inevitably contains approximations, in particular the quark and chromon model in more detail. We first provide more argument for the quark and chromon model. In the constituent gluon model, the gauge-invariant combinations of the octet gluons, $g \bar{g}$ or $g g g$, have been thought to form the 
glueballs. This is because all gluons are treated equally in this model.

As we have pointed out, this has a critical defect. Obviously, an important role of gluons is to provide the binging force of the colored objects. So, if all gluons become the constituent, it is very difficult to explain how they provide the binding. In the quark and chromon model, however, only the chromons become the constituent gluon. This is because only the chromons carry the color charge. In comparison, the neurons, being color neutral, naturally assume the role of the binding gluons.

To clarify this point, we compare the possible Feynman diagrams of two and three chromon interactions shown in Fig. 5 with the similar Feynman diagrams of neuron interactions shown in Fig. 6. Clearly, Fig. 5 looks very similar to the Feynman diagrams of $q \bar{q}$ and $q q q$ bound states in the quark model. This means that the chromons, just like the quarks, can become the constituents of hadrons. In particular, this means that they could form chromoball bound states among themselves.

On the other hand, Fig. 6 looks totally different from Fig. 5. Obviously, Fig. 6 looks very much like the photon self-interaction in QED. This is because the neurons are not colored, so that they can interact only through the chromon or quark loops. So they actually play the role of "the photons" in QCD whose binding is much weaker than the chromon binding. This strongly support the quark and chromon model.

Of course, although the photons in QED do not form a bound state, there is still a possibility that the neuron binding is strong enough to form a bound state in QCD. Nevertheless, it is natural to assume that if the neurons form a bound state at all, they should form a very weakly bound state which would look like a bound state of two quarkoniums or a molecular state made of two light mesons. This means that there could be only a few neuroballs, the bound states made of neurons, maybe one or two at most. For this reason, we will assume that only the chromons become the constituent in this paper.

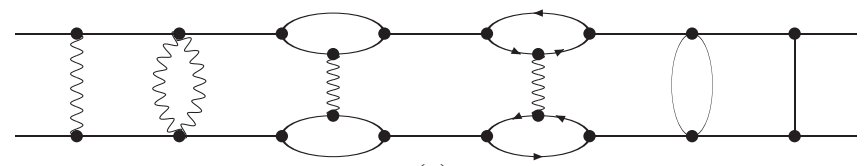

(a)

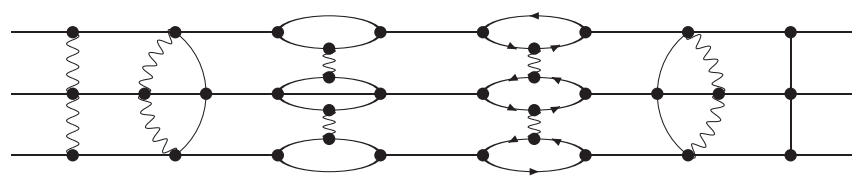

(b)

FIG. 5. The possible Feynman diagrams which bind the chromons. Two chromon binding is shown in (A), three chromon binding is shown in (B). The quarks are represented by the arrows.

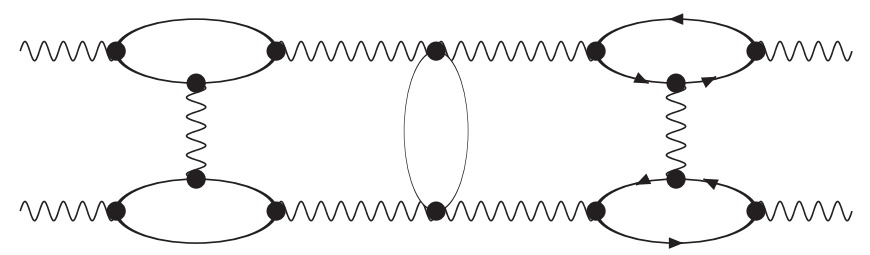

(a)

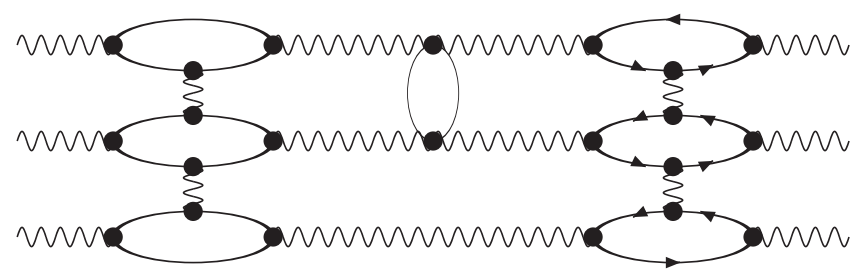

(b)

FIG. 6. The possible Feynman diagrams of the neuron interaction. Two neuron binding is shown in (A), three neuron binding is shown in (B). The quarks are represented by the arrows.

This, however, does not mean that they cannot contribute to the binding. Clearly, Figs. 5 and 6 show that both neurons and chromons can exchange chromons to make the binding. However, we emphasize that there is a clear difference between the role of the chromon to be the constituent gluon and the exchange gluon.

Now we discuss the characteristic features and new predictions of the quark and chromon model, and show how we can test the model experimentally. As we have explained, the most important change in this model is the replacement of the non-Abelian gauge group by the color reflection group. Indeed, the color reflection symmetry becomes the backbone of the quark and chromon model $[22,23,32]$.

This simplifies the non-Abelian gauge invariance to the color reflection invariance. So only the color reflectioninvariant combinations of the chromons become gauge invariant and thus form the glueballs. This is why the color reflection group becomes so important in this model.

To amplify this point, we emphasize that the model reclassifies all hadrons in the quark model. For example, in the quark model mesons and baryons are viewed as color singlets made of $(3 \times \overline{3})$ and $(3 \times 3 \times 3) \mathrm{SU}(3)$ quark triplets. However, in the new model, the quark triplets should be interpreted as the triplet of the color reflection group, not the color $\mathrm{SU}(3)$. So, in this reclassification the mesons and baryons acquire a different interpretation. This, of course, would not change the hadron spectrum much. However, it clearly shows that the quark and chromon model sheds a new light on the old quark model, even for the hadrons made of quarks.

Notice that for the meson classification the full color reflection symmetry becomes important, because the antichromons become an important ingredient. However, for 
the baryons, the Weyl symmetry plays the main role because baryons have only quarks, not antiquarks.

Obviously, a place where the quark and chromon model makes a big difference is in the glueball and hybrid hadron. The model asserts that only the chromoball, the bound state of chromons, can become the glueball. In other words, the $g g$ and $g g g$ glueballs are actually the color singlets made of $(6 \times \overline{6})$ and $(6 \times 6 \times 6)$ sextet chromons of the color reflection group, not $(8 \times \overline{8})$ and $(8 \times 8 \times 8) \mathrm{SU}(3)$ octets. This is the difference between the quark and chromon model and the constituent gluon model $[7,8]$. So in this model the quark triplet and chromon sextet of the color reflection group become the essential ingredients of hadrons.

As importantly the model provides conceptually a clear picture of chromoball mixing with the quarkonium. Moreover, the model predicts the existence of the hybrid hadrons made of the quark triplets and chromon sextets. So studying the chromoballs and their mixing with quarkoniums and the hybrid hadrons predicted by the model we can test the quark and chromon model.

In the quark and chromon model, one expects infinite tower chromoballs, but experimentally we have not so many candidates of glueballs. There could be two possible explanations for this. First, they could easily mix with quakoniums, unless the conservation of quantum number forbids the mixing. This means that in reality the physical glueballs are mixed states, not pure chromoball states. Certainly this makes the experimental identification of the glueballs a nontrivial matter [32].

Second, the chromoballs may have an intrinsic instability and decay faster than ordinary hadrons, which could make the experimental identification difficult. As we have pointed out, the chromons tend to annihilate each other in the color background, which has to do with the antiscreening of the color charge [30-32,38-40].

We can estimate the glueball partial decay width coming from this instability. According to the QCD one-loop effective action, the chromon annihilation probability per unit volume per unit time is given by [30-32]

$$
\Gamma_{A}=\sum_{p} \frac{11 g^{2}}{96 \pi} \bar{E}_{p}^{2} \times \frac{4 \pi}{3 \Lambda_{\mathrm{QCD}}^{3}},
$$

where the sum is on three $\mathrm{SU}(2)$ subgroups and $\bar{E}_{p}$ is the average chromo-electric field of each subgroup inside the glueballs. Now, if we choose $\alpha_{s} \simeq 0.4, \Lambda_{\mathrm{QCD}} \simeq 339 \mathrm{MeV}$ (for three quark flavors), and $\bar{E}_{p} \simeq(g / \pi) \Lambda_{\mathrm{QCD}}^{2}$ we have $\Gamma_{A} \simeq 398 \mathrm{MeV}$ [12]. However, notice that with $\Lambda_{\mathrm{QCD}} \simeq 200 \mathrm{MeV}$, we have $\Gamma_{A} \simeq 235 \mathrm{MeV}$ [51].

Of course, this is a rough estimate which depends on many things. For example, the $g \bar{g}$ glueballs and $g g g$ glueballs may have different color field strengths and different sizes, and thus may have a different lifetime. However, we emphasize that the above estimate is the partial decay width we expect from the asymptotic freedom, in addition to the "normal" hadronic decay width. This strongly implies that in general the glueballs (in particular excited ones) are expected to have very short lifetime.

This instability has another important implication. It has been widely believed that "the gluon condensation" plays important role in QCD dynamics [6]. However, the gluon pair annihilation shown in (34) strongly suggests that this gluon condensation should become unstable, and thus cannot last. Moreover, QCD already has the monopole condensation. This makes the gluon condensation highly improbable.

\section{GLUEBALL-QUARKONIUM MIXING}

In the preceding paper, we have outlined how the chromoballs can mix with quarkoniums in the quark and chromon model, to show the viability of the above theoretical discussions [32]. In this paper, we discuss the mixing in more detail. The possible Feynman diagrams for the mixing are shown in Fig. 7, which tells that the mixing takes place not just between chromoballs and quarkoniums but also between the $c c$ and $c c c$ chromoballs, directly or through the virtual states made of neurons and/or molecular bound states of mesons. So in the mixing diagram the role of neuron and chromon is blurred.

Obviously, the mixing influences the $q \bar{q}$ octet-singlet mixing in the quark model. So we review the octet-singlet mixing in the quark model first. Let

$$
\begin{aligned}
\langle u \bar{u}|H| u \bar{u}\rangle_{E x} & =\langle d \bar{d}|H| d \bar{d}\rangle_{E x}=E, \\
\langle s \bar{s}|H| s \bar{s}\rangle_{E x} & =E^{\prime}=E+\Delta, \\
\left\langle q^{\prime} \bar{q}^{\prime}|H| q \bar{q}\right\rangle_{A n} & =A, \quad\left(\text { for all } \mathrm{q}, \mathrm{q}^{\prime}\right) .
\end{aligned}
$$

Now with

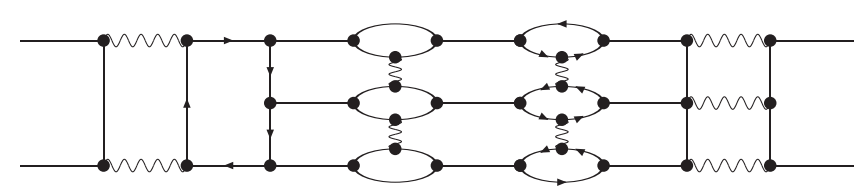

(a)

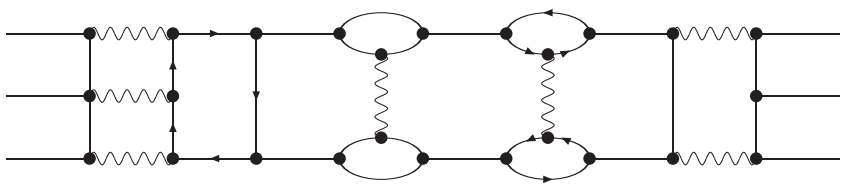

(b)

FIG. 7. The possible glueball-quarkonium mixing diagrams. The $g g$ and $g g g$ chromoball mixing with quarkoniums are shown in (A) and (B). 


$$
\begin{aligned}
& |8\rangle=\frac{|u \bar{u}\rangle+|d \bar{d}\rangle-2|s \bar{s}\rangle}{\sqrt{6}}, \\
& |1\rangle=\frac{|u \bar{u}\rangle+|d \bar{d}\rangle+|s \bar{s}\rangle}{\sqrt{3}},
\end{aligned}
$$

we may obtain the following mass matrix for the $q \bar{q}$ which describes the octet-singlet mixing [32],

$$
\begin{aligned}
M^{2} & =\left(\begin{array}{cc}
\langle 8|H| 8\rangle & \langle 8|H| 1\rangle \\
\langle 1|H| 8\rangle & \langle 1|H| 1\rangle
\end{array}\right) \\
& =\left(\begin{array}{cc}
E+\frac{2}{3} \Delta & -\frac{\sqrt{2}}{3} \Delta \\
-\frac{\sqrt{2}}{3} \Delta & E+\frac{1}{3} \Delta+3 A
\end{array}\right) .
\end{aligned}
$$

Notice that $\Delta$-term is responsible for the mixing. However, we emphasize that this mixing among the quarks cannot provide the correct octet-singlet mixing because the glueballs inevitably influence the quark octet-singlet mixing. This is evident in Fig. 7.

Clearly, we can generalize (37) to the following $3 \times 3$ mixing matrix of one lightest chromoball $|G\rangle$ with the quark nonet [32]

$$
M^{2}=\left(\begin{array}{ccc}
E+\frac{2}{3} \Delta & -\frac{\sqrt{2}}{3} \Delta & 0 \\
-\frac{\sqrt{2}}{3} \Delta & E+\frac{1}{3} \Delta+3 A & \nu \\
0 & \nu & G
\end{array}\right) .
$$

Of course, a similar mixing matrix has been used in the constituent gluon model. As we have pointed out, however, this model has critical defects. Our quark and chromon model can be viewed as a new model which justifies the above mixing without such defects.

In principle, we should be able to calculate the parameters in the mass matrix theoretically. For example, we could calculate $G$ using the gauge-invariant current operator, or calculate the mixing parameter $\nu$ using the Feynman diagrams in our model. However, in this paper, we will fix the parameters with experimental data to see how well the mixing matrix can explain the glueball-quarkonium mixing.

Now, diagonalizing the mass matrix, we can transform the unphysical states $(|8\rangle,|1\rangle,|G\rangle)$ to the mass eigenstates $\left(\left|m_{1}\right\rangle,\left|m_{2}\right\rangle,\left|m_{3}\right\rangle\right)$, and obtain the information on the chromon and quark contents of the physical states. Notice that, assuming that after the confinement the chromons acquire the constituent mass $\mu$, we can put $G=4 \mu^{2}$ (supposing the chromoball mass before the mixing is given by $\sqrt{G}=2 \mu$ ).

We can easily generalize the mass matrix to the $4 \times 4$ mixing

$$
M^{2}=\left(\begin{array}{cccc}
E+\frac{2}{3} \Delta & -\frac{\sqrt{2}}{3} \Delta & 0 & 0 \\
-\frac{\sqrt{2}}{3} \Delta & E+\frac{1}{3} \Delta+3 A & \nu & \nu^{\prime} \\
0 & \nu & G & \epsilon \\
0 & \nu^{\prime} & \epsilon & G^{\prime}
\end{array}\right),
$$

to include one more chromoball state $\left|G^{\prime}\right\rangle$. This has eight parameters, but we may reduce the parameters to seven by diagonalizing the $2 \times 2$ chromoball mass matrix first and putting $\epsilon=0$. With this we can express $G$ and $G^{\prime}$ by the chromon mass $\mu$ and put $G=4 \mu^{2}$ and $G^{\prime}=4 \mu^{2}+\delta$ for two chromon bound state or $G^{\prime}=9 \mu^{2}+\delta$ for three chromon bound state. Clearly, (38) and (39) demonstrate that the chromoball-quarkonium mixing inevitably influences the quarkonium octet-singlet mixing. So we cannot discuss the $q \bar{q}$ spectroscopy without the chromoballs.

Diagonalizing the mass matrix we can figure out the quark and chromon contents of the mass eigenstates. Moreover, knowing the chromon content of the physical states, we can calculate the relative branching ratios of iso-singlet mesons made of heavy quarks, e. g., the $J / \psi$ radiative decay to the physical states in each channel. This is because these decays are the Okubo-Zweig-Iizuka (OZI) suppressed process which can only be made possible through the intermediate chromoball states.

Let $\alpha_{i}$ be the parameters of the mixing matrix which determine the gluon content of physical states $\left|m_{i}\right\rangle$. We can predict the relative branching ratios of $J / \psi$ to $\gamma X$ decays among the physical states with $\alpha_{i}$, because these decays are induced by gluons. So, for the $\mathrm{S}$ wave decay (i.e., for $0^{++}$ and $2^{++}$) we have [32]

$$
R\left(\frac{J / \psi \rightarrow \gamma X_{k}}{J / \psi \rightarrow \gamma X_{i}}\right)=\left(\frac{\alpha_{k}}{\alpha_{i}}\right)^{2}\left(\frac{m_{\psi}^{2}-m_{k}^{2}}{m_{\psi}^{2}-m_{i}^{2}}\right)^{3},
$$

but for the $\mathrm{P}$ wave decay (i.e., for $0^{-+}$), we expect to have

$$
R\left(\frac{J / \psi \rightarrow \gamma X_{k}}{J / \psi \rightarrow \gamma X_{i}}\right)=\left(\frac{\alpha_{k}}{\alpha_{i}}\right)^{2}\left(\frac{m_{\psi}^{2}-m_{k}^{2}}{m_{\psi}^{2}-m_{i}^{2}}\right)^{5},
$$

where the last term is the kinematic phase space factor. Clearly, this argument can also be applied to similar OZI suppressed decays of heavy $t \bar{t}$ or $b \bar{b}$ iso-singlet mesons.

It must be pointed out that, although the chromoballs in general mix with the quarkoniums, in particular cases the pure chromoballs could exist. This is because some of the $g g$ chromoballs become the oddballs, which have the quantum number $J^{P C}$ that $q \bar{q}$ cannot have, and thus cannot mix with the quarkoniums [7,32]. Obviously, these lowlying oddballs become very important for us to search for the pure chromoballs.

Independent of the details, however, we emphasize the clarity of the mixing mechanism in our quark and chromon model. All terms in the mass matrix have clear physical 
meaning. For example, we can draw the Feynman diagram which represents the parameter $\nu$ in (38), and could in principle calculate it theoretically.

Before we close this section, it is worth comparing our model with the so-called "model-independent" calculations in the conventional QCD. First, let us compare our model with the QCD sum rule approach which uses the gaugeinvariant current operator to calculate the glueball mass, which has been asserted to be model independent [6]. Here, they calculate the mass of the scalar glueball from the simplest gauge-invariant $0^{++}$current operator $\left\langle\vec{F}_{\mu \nu} \cdot \vec{F}_{\mu \nu}\right\rangle$ which supposedly describes the glueball made of two gluons. Similarly, for the $2^{++}$glueball they have $\left\langle\vec{F}_{\mu \alpha} \cdot \vec{F}_{\alpha \nu}\right\rangle$. However, notice that actually these operators contain two, three, and four gluons, so that it is difficult to justify them as two gluon states.

On the other hand, in our model, the simplest $0^{++}$and $2^{++}$current operators are just two chromon states given by $\left\langle\vec{X}_{\mu} \cdot \vec{X}_{\mu}\right\rangle$ and $\left\langle\vec{X}_{\mu} \cdot \vec{X}_{\nu}\right\rangle$ [21-23]. Similarly, for the glueballs made of three chromons we have $\left\langle d_{a b c} X_{\mu}^{a} X_{\nu}^{b} X_{\rho}^{c}\right\rangle$ and $\left\langle f_{a b c} X_{\mu}^{a} X_{\nu}^{b} X_{\rho}^{c}\right\rangle$. This is simply impossible in the conventional QCD.

Exactly the same thing can be said about the lattice calculation. Here, again, the calculations are often claimed to be "model independent." However, once we understand the hidden structures of QCD we have much simpler ways to calculate the physical quantities. So the conventional "model-independent" calculations simply become obsolete and old fashioned after the Abelian decomposition provides new and simpler ways to calculate the physical quantities. This is the advantage of the Abelian decomposition.

\section{NUMERICAL ANALYSIS}

The above discussion shows that the mixing analysis is a crucial step for us to identify the glueballs. For the $3 \times 3$ mixing the mass matrix has five parameters, but we can fix $E$ and $\Delta$ from the $q \bar{q}$ flavor octet data. So we need three inputs to fix the mass matrix completely. There are different ways to fix them. One way is to choose two mass eigenstates from PDG and treat $G$ (or equivalently the chromon mass $\mu$ ) as a free parameter, and find the best fit for $\mu$ which could predict the third physical state and explain the PDG data best. Another way is to use all three mass eigenstates as the input, and determine the chromon mass as well.

For the $4 \times 4$ mixing the matrix has seven parameters, but we can reduce this number to five fixing two of them from the $q \bar{q}$ flavor octet data. With this we may choose four mass eigenstates as the input (when available) and find the physical contents of the mass eigenstates, treating the chromon mass as free parameters. Or we may choose three mass eigenstates as the input and predict the mass of the fourth physical state, imposing an extra constraint, e.g., $\nu^{\prime}=\nu$ etc.
In the preceding paper, we have discussed the numerical analysis of the mixing below $2 \mathrm{GeV}$ in $0^{++}, 2^{++}$and $0^{-+}$ channels with this strategy [32]. However, the numerical analysis was preliminary and inconclusive, partly because it depends very much on how to choose the inputs. In the following, we discuss the mixing in more detail, and improve the results of the preceding paper.

\section{A. $0^{++}$channel}

In this channel, PDG lists five iso-singlet mesons, $f_{0}(500), f_{0}(980), f_{0}(1370), f_{0}(1500)$, and $f_{0}(1710)$ below $2 \mathrm{GeV}$ [12]. However, the interpretation of the scalar mesons has been difficult and controversial, because some of them have unusually large decay width and some of them could be viewed as non- $q \bar{q}$ multiquark states [13-15]. In this paper, we try to figure out their physical content within the quark and chromon model with the following two important issues in mind.

The first issue is what should we choose to be the isotriplet partner of the flavor octet in this channel. This is very important because this determines the inputs $E$ and $\Delta$ in the mixing analysis. PDG suggests that the flavor octet partner of the $0^{++}$isosinglet are $a_{0}(1450)$ and $K_{0}^{*}(1430)$, not $a_{0}(980)$ and $K_{0}^{*}(1430)$ [12]. Intuitively, this looks somewhat strange because this implies that the $u$ and $d$ quarks are heavier (or at least not lighter) than the $s$ quark. So it is worth for us to study the possibility that $a_{0}(980)$ and $K_{0}^{*}(1430)$ become the octet partners.

If we adopt the PDG view and identify $a_{0}(1450)$ to be the isotriplet partner, we may choose [12]

$$
\begin{aligned}
& E=m^{2}\left(a_{0}\right), \quad a_{0}=a_{0}(1450), \\
& \Delta=2\left(m^{2}(K)-m^{2}\left(a_{0}\right)\right), \quad K=K_{0}^{*}(1430) .
\end{aligned}
$$

as the input. However, as we have remarked, it is worth for us to check whether this PDG view is correct or not. Since the strange meson of the flavor octet of this channel is $K_{0}^{*}(1430)$, one would expect the mass of the nonstrange isotriplet partner to be less than $1430 \mathrm{MeV}$.

In this case, $a_{0}(980)$ becomes a natural candidate of the isotriplet partner of the flavor octet, and we may choose

$$
\begin{aligned}
& E=m^{2}\left(a_{0}\right), \quad a_{0}=a_{0}(980), \\
& \Delta=2\left(m^{2}(K)-m^{2}\left(a_{0}\right)\right), \quad K=K_{0}^{*}(1430) .
\end{aligned}
$$

as the input. So we have two possible inputs, (42) and (43).

The second issue is the interpretation of $f_{0}(500)$, which has an unusually broad decay width. According to PDG it does not fit to the quark model well, and there have been suggestions that it could be either a tetra-quark state or a mixed state [52-58]. However, there are other logical possibilities.

First, it could be viewed as a neuroball, the glueball made of neurons [32]. As we pointed out, the neurons (just like the 
TABLE I. The numerical analysis of the $3 \times 3$ mixing in the $0^{++}$channel, with $a_{0}(1450), f_{0}(1500)$, and $f_{0}(1710)$ as the input. Here, the third physical state can be identified as $f_{0}(1370)$.

\begin{tabular}{|c|c|c|c|c|c|c|c|c|c|c|c|c|c|c|}
\hline \multirow[b]{2}{*}{$\mu$} & \multirow[b]{2}{*}{$A$} & \multirow[b]{2}{*}{$\nu$} & \multirow[b]{2}{*}{$m_{3}$} & \multicolumn{3}{|c|}{$m_{1}=f_{0}(1500)$} & \multicolumn{3}{|c|}{$m_{2}=f_{0}(1710)$} & \multicolumn{3}{|c|}{$m_{3}$} & \multirow[b]{2}{*}{$R\left(m_{2} / m_{1}\right)$} & \multirow[b]{2}{*}{$R\left(m_{3} / m_{1}\right)$} \\
\hline & & & & $u+d$ & $s$ & $G$ & $u+d$ & $s$ & $G$ & $u+d$ & $s$ & $G$ & & \\
\hline 0.76 & 0.27 & 0.18 & 1.40 & 0.07 & 0.00 & 0.93 & 0.73 & 0.20 & 0.07 & 0.19 & 0.80 & 0.00 & 0.05 & 0.00 \\
\hline 0.78 & 0.23 & 0.31 & 1.40 & 0.26 & 0.01 & 0.73 & 0.59 & 0.16 & 0.25 & 0.15 & 0.83 & 0.02 & 0.14 & 0.02 \\
\hline 0.80 & 0.18 & 0.36 & 1.39 & 0.44 & 0.01 & 0.54 & 0.45 & 0.12 & 0.43 & 0.11 & 0.87 & 0.02 & 0.59 & 0.05 \\
\hline 0.82 & 0.14 & 0.35 & 1.39 & 0.62 & 0.02 & 0.36 & 0.30 & 0.08 & 0.62 & 0.09 & 0.90 & 0.01 & 1.26 & 0.07 \\
\hline 0.84 & 0.09 & 0.29 & 1.39 & 0.79 & 0.02 & 0.18 & 0.15 & 0.04 & 0.80 & 0.05 & 0.93 & 0.01 & 3.26 & 0.09 \\
\hline 0.86 & 0.04 & 0.07 & 1.39 & 0.96 & 0.03 & 0.01 & 0.01 & 0.00 & 0.99 & 0.03 & 0.97 & 0.00 & 85.71 & 0.12 \\
\hline
\end{tabular}

photons in QED) have very weak binding because they can interact only through the quark or chromon loops. Nevertheless, they could form a loosely bound state which has a broad decay width. And this is exactly what we find in $f_{0}(500)$. This is in line with the popular interpretation that $f_{0}(500)$ is a tetra-quark state [52-58]. This is evident in Fig. 6, where the loops can be viewed as $q \bar{q}$ or $g g$ bound states.

Another possibility is that $f_{0}(500)$ could be the monoball, the vacuum fluctuation mode of the monopole condensation, in QCD [32]. As we have pointed out, if the color confinement comes from the monopole condensation, QCD could have a $0^{++}$vacuum fluctuation mode [21,22]. In this case, $f_{0}(500)$ becomes a natural candidate of this vacuum fluctuation. This suggests that $f_{0}(500)$ may not be a simple chromoball or $q \bar{q}$ state.

With this in mind, we can discuss the mixing. Let us first exclude $f(500)$ in the mixing for the reason discussed above. In the preceding paper, we have discussed the $3 \times 3$ mixing with $f_{0}(1500)$ and $f_{0}(1710)$ as the input, adopting the PDG view (42) [32]. The result is copied in Table I. Notice that the table shown in the preceding paper had a typological mistake that the numbers in the last two columns (i.e., $R\left(m_{2} / m_{1}\right)$ and $R\left(m_{3} / m_{1}\right)$ ) were interchanged. This mistake is corrected in Table I.

The table shows that the third state (with mass around $1400 \mathrm{MeV}$ ) could be identified to be $f_{0}(1370)$, which becomes predominantly an $s \bar{s}$ state. However, the physical contents of two other states depend very much on the value of the chromon mass parameter $\mu$. When $\mu$ is around $760 \mathrm{MeV}$, $f_{0}(1500)$ become predominantly a chromoball state and $f_{0}(1710)$ becomes predominantly the $u \bar{u}+d \bar{d}$ state.
On the other hand, when $\mu$ increases to $860 \mathrm{MeV}$, $f_{0}(1500)$ becomes a $u \bar{u}+d \bar{d}$ state and $f_{0}(1710)$ quickly becomes a chromoball state. However, $f_{0}(1370)$ remains to be the $s \bar{s}$ state, so that here the $s \bar{s}$ state becomes lighter than the $u \bar{u}+d \bar{d}$ state. This, of course, is due to the input (42). This is against the PDG interpretation, which suggests that $f_{0}(1370)$ is the $u \bar{u}+d \bar{d}$ state and $f_{0}(1710)$ is the $s \bar{s}$ state.

On the other hand, if we adopt (43) as the input, we obtain Table II. Here, we have chosen $f_{0}(980)$ and $f_{0}(1500)$ as the input. The result shows that when $\mu \simeq 750 \mathrm{MeV}$, the third state has mass around $1800 \mathrm{MeV}$ and could be identified as $f_{0}(1710)$. In this case, $f_{0}(1500)$ remains predominantly a chromoball state, but $f_{0}(1710)$ becomes predominantly the $s \bar{s}$ state and $f_{0}(980)$ becomes predominantly the $u \bar{u}+d \bar{d}$ state. This, of course, is what we have expected from (43).

Clearly, the two tables give different descriptions, and we have to know which is closer to the truth. One way to find which is better is to compare the predictions of the relative radiative decay ratios of $J / \psi$ with the experimental data. Experimentally, PDG has new data on the radiative decay of $J / \psi$ to $0^{++}$states [12],

$$
J / \psi \rightarrow \gamma f_{0}(1710) \rightarrow\left\{\begin{array}{l}
\gamma K \bar{K} \simeq(8.5+1.2-0.9) \\
\times 10^{-4} \\
\gamma \pi \pi \simeq(4.0 \pm 1.0) \times 10^{-4} \\
\gamma \omega \omega \simeq(3.1 \pm 1.0) \times 10^{-4} \\
\gamma \eta \eta \simeq(2.4+1.2-0.7) \\
\times 10^{-4}
\end{array}\right.
$$

TABLE II. The numerical analysis of the $3 \times 3$ mixing in the $0^{++}$channel, with $a_{0}=a_{0}(980), f_{0}(980)$, and $f_{0}(1500)$ as the input. Here, the third physical state could be identified as $f_{0}(1710)$.

\begin{tabular}{|c|c|c|c|c|c|c|c|c|c|c|c|c|c|c|}
\hline \multirow[b]{2}{*}{$\underline{\mu}$} & \multirow[b]{2}{*}{$A$} & \multirow[b]{2}{*}{$\nu$} & \multirow[b]{2}{*}{$m_{3}$} & \multicolumn{3}{|c|}{$m_{1}=f_{0}(980)$} & \multicolumn{3}{|c|}{$m_{2}=f_{0}(1500)$} & \multicolumn{3}{|c|}{$m_{3}$} & \multirow[b]{2}{*}{$R\left(m_{2} / m_{1}\right)$} & \multirow[b]{2}{*}{$R\left(m_{3} / m_{1}\right)$} \\
\hline & & & & $u+d$ & $s$ & $G$ & $u+d$ & $s$ & $G$ & $u+d$ & $s$ & $G$ & & \\
\hline 0.55 & 2.44 & 1.29 & 3.06 & 0.05 & 0.00 & 0.95 & 0.44 & 0.54 & 0.02 & 0.51 & 0.46 & 0.02 & 0.01 & 0.00 \\
\hline 0.60 & 1.91 & 1.62 & 2.83 & 0.11 & 0.00 & 0.89 & 0.43 & 0.52 & 0.05 & 0.46 & 0.48 & 0.06 & 0.04 & 0.00 \\
\hline 0.65 & 1.33 & 1.68 & 2.55 & 0.21 & 0.00 & 0.79 & 0.40 & 0.49 & 0.11 & 0.39 & 0.51 & 0.10 & 0.09 & 0.01 \\
\hline 0.70 & 0.71 & 1.44 & 2.21 & 0.41 & 0.00 & 0.59 & 0.34 & 0.42 & 0.24 & 0.24 & 0.58 & 0.17 & 0.26 & 0.05 \\
\hline 0.75 & 0.04 & 0.36 & 1.79 & 0.95 & 0.00 & 0.05 & 0.05 & 0.06 & 0.89 & 0.00 & 0.94 & 0.05 & 10.75 & 0.43 \\
\hline
\end{tabular}


and

$$
J / \psi \rightarrow \gamma f_{0}(1500) \rightarrow\left\{\begin{array}{l}
\gamma \pi \pi \simeq(1.01 \pm 0.32) \times 10^{-4} \\
\gamma \eta \eta \simeq(1.7+0.6-1.4) \\
\times 10^{-5}
\end{array}\right.
$$

Of course, this may not be the final data, because other decay modes could be discovered later. However, assuming that this is the final result, we have

$$
R\left(f_{0}(1710) / f_{0}(1500)\right) \simeq 15.3 .
$$

This is a very important piece of information, because this determines the glue content of the mass eigenstates.

Now, Table II predicts the relative radiative decay ratio to be $R\left(f_{0}(1710) / f_{0}(1500)\right) \simeq 0.04 \quad$ (at $\left.\mu=750 \mathrm{MeV}\right)$. Clearly, this is not in line with (44), which is troublesome. In comparison, according to Table I we have $R\left(f_{0}(1710) /\right.$ $\left.f_{0}(1500)\right) \simeq 15.3($ at $\mu \simeq 856 \mathrm{MeV})$. This is in good agreement with the PDG data, which implies that Table I is better. This in turn implies that $a_{0}(1450)$, not $a_{0}(980)$, could be the isotriplet partner of these isosinglet states. However, this conclusion is premature because the contents of physical states in Table I is controversial and $R\left(m_{2} / m_{1}\right)$ becomes very sensitive to the change of $\mu$.

Independent of whether this result is correct or not, however, the above $3 \times 3$ mixing has a critical shortcoming in that it can explain the mixing of only three physical states, while here we have at least four physical states (excluding $f_{0}(500)$ ) below $2 \mathrm{GeV}$. This strongly motivates us to go to the $4 \times 4$ mixing. And this is independent of which input, (42) or (43), we choose.

So we consider the $4 \times 4$ mixing (39) with two chromoball states ${ }^{1} S_{0}$ and ${ }^{5} D_{0}$ made of two chromons $|G\rangle$ and $\left|G^{\prime}\right\rangle$.
Diagonalizing the two chromoball mass matrix first, we may put

$$
\epsilon=0, \quad G=4 \mu^{2}, \quad G^{\prime}=G+\delta,
$$

and consider the mixing of the two $q \bar{q}$ states with two chromoballs which have mass $\sqrt{G}$ and $\sqrt{G^{\prime}}$. This has seven parameters, but we can fix two with (42) or (43) and four with the four mass eigenstates $f_{0}(980), f_{0}(1370)$, $f_{0}(1500)$, and $f_{0}(1710)$ as the input. With this, we can diagonalize the mass matrix and find the physical contents of the mass eigenstates, treating the chromon mass $\mu$ as the free parameter.

Now, adopting the PDG view (42) we obtain Table III, but with (43) we obtain Table IV. However, the mathematical equations which we need to solve to diagonalize the mass matrix are very rigid which often have no solution, and this forces us to change the input data slightly to find the solutions. So here we have changed the four mass eigenstates to $990,1400,1505$, and $1722 \mathrm{MeVs}$ to obtain Table III, and to $990,1370,1505,1800 \mathrm{MeVs}$ to obtain Table IV.

The numerical result of Table III obtained with (42) suggests that $f_{0}(980)$ is predominantly the ${ }^{1} S_{0}$ chromoball state and $f_{0}(1370)$ is predominantly the $s \bar{s}$ state. However, $f_{0}(1500)$ becomes largely the ${ }^{5} D_{0}$ chromoball state and $f_{0}(1710)$ becomes largely the $u \bar{u}+d \bar{d}$ state, although they have considerable mixing as the chromon mass increases to $600 \mathrm{MeV}$. This is in line with Table I. However, here the $u \bar{u}+d \bar{d}$ state remains heavier than the $s \bar{s}$ state, which again is due to the input (42).

On the other hand, Table IV obtained with (43) tells that $f_{0}(980)$ and $f_{0}(1710)$ are the $u \bar{u}+d \bar{d}$ and $s \bar{s}$ states, respectively. And $f_{0}(1370)$ and $f_{0}(1500)$ become the

\begin{tabular}{|c|c|c|c|c|c|c|c|c|c|c|c|c|c|c|c|c|}
\hline \multirow[b]{2}{*}{$\mu$} & \multicolumn{4}{|c|}{$m_{1}=f_{0}(980)$} & \multicolumn{4}{|c|}{$m_{2}=f_{0}(1370)$} & \multicolumn{4}{|c|}{$m_{3}=f_{0}(1500)$} & \multicolumn{4}{|c|}{$m_{4}=f_{0}(1710)$} \\
\hline & $u+d$ & $s$ & $G$ & $G^{\prime}$ & $u+d$ & $s$ & $G$ & $G^{\prime}$ & $u+d$ & $s$ & $G$ & $G^{\prime}$ & $u+d$ & $s$ & $G$ & $G^{\prime}$ \\
\hline 0.50 & 0.01 & 0.01 & 0.99 & 0.00 & 0.19 & 0.81 & 0.00 & 0.01 & 0.13 & 0.00 & 0.00 & 0.86 & 0.68 & 0.18 & 0.01 & 0.13 \\
\hline 0.52 & 0.03 & 0.03 & 0.94 & 0.00 & 0.18 & 0.80 & 0.01 & 0.01 & 0.17 & 0.01 & 0.02 & 0.81 & 0.62 & 0.17 & 0.04 & 0.17 \\
\hline 0.54 & 0.06 & 0.05 & 0.88 & 0.01 & 0.18 & 0.79 & 0.01 & 0.01 & 0.21 & 0.01 & 0.04 & 0.74 & 0.55 & 0.15 & 0.06 & 0.24 \\
\hline 0.56 & 0.09 & 0.08 & 0.82 & 0.01 & 0.18 & 0.79 & 0.02 & 0.01 & 0.26 & 0.01 & 0.08 & 0.65 & 0.47 & 0.13 & 0.08 & 0.33 \\
\hline 0.58 & 0.13 & 0.11 & 0.75 & 0.01 & 0.18 & 0.78 & 0.03 & 0.01 & 0.32 & 0.01 & 0.14 & 0.53 & 0.38 & 0.10 & 0.08 & 0.44 \\
\hline 0.60 & 0.17 & 0.14 & 0.67 & 0.02 & 0.17 & 0.77 & 0.05 & 0.01 & 0.38 & 0.01 & 0.22 & 0.39 & 0.27 & 0.07 & 0.07 & 0.58 \\
\hline$\mu$ & \multicolumn{3}{|c|}{$R\left(m_{2} / m_{1}\right)$} & \multicolumn{3}{|c|}{$R\left(m_{3} / m_{1}\right)$} & \multicolumn{2}{|c|}{$R\left(m_{4} / m_{1}\right)$} & \multicolumn{3}{|c|}{ A } & $\nu$ & \multicolumn{3}{|c|}{$\nu^{\prime}$} & $\delta$ \\
\hline 0.50 & \multicolumn{3}{|c|}{0.01} & \multicolumn{3}{|c|}{0.54} & \multicolumn{2}{|r|}{0.06} & \multicolumn{3}{|c|}{0.25} & 0.19 & \multicolumn{3}{|c|}{0.24} & 1.35 \\
\hline 0.52 & \multicolumn{3}{|c|}{0.01} & \multicolumn{3}{|c|}{0.54} & \multicolumn{2}{|r|}{0.10} & \multicolumn{3}{|c|}{0.21} & 0.41 & \multicolumn{3}{|c|}{0.28} & 1.30 \\
\hline 0.54 & \multicolumn{3}{|c|}{0.02} & \multicolumn{3}{|c|}{0.54} & \multicolumn{2}{|r|}{0.16} & \multicolumn{3}{|c|}{0.17} & 0.53 & \multicolumn{3}{|c|}{0.32} & 1.26 \\
\hline 0.56 & \multicolumn{3}{|c|}{0.03} & \multicolumn{3}{|c|}{0.54} & \multicolumn{2}{|r|}{0.22} & \multicolumn{3}{|c|}{0.13} & 0.61 & \multicolumn{3}{|c|}{0.36} & 1.22 \\
\hline 0.58 & \multicolumn{3}{|c|}{0.04} & \multicolumn{3}{|c|}{0.54} & \multicolumn{2}{|r|}{0.31} & & 0.07 & & 0.66 & & 0.40 & & 1.20 \\
\hline 0.60 & & 0.0 & & & 0.54 & & & 0.43 & & 0.01 & & 0.68 & & 0.42 & & 1.21 \\
\hline
\end{tabular}

TABLE III. The numerical analysis of the $4 \times 4$ mixing in the $0^{++}$channel, with $f_{0}(980), f_{0}(1370), f_{0}(1500)$, and $f_{0}(1710)$ as the input. Here, $a_{0}(1450)$ is identified as the isotriplet partner. 
TABLE IV. The numerical analysis of the $4 \times 4$ mixing in the $0^{++}$channel, with $f_{0}(980), f_{0}(1370), f_{0}(1500)$, and $f_{0}(1710)$ as the input. Here, $a_{0}(980)$ is identified as the isotriplet partner.

\begin{tabular}{|c|c|c|c|c|c|c|c|c|c|c|c|c|c|c|c|c|}
\hline \multirow[b]{2}{*}{$\mu$} & \multicolumn{4}{|c|}{$m_{1}=f_{0}(980)$} & \multicolumn{4}{|c|}{$m_{2}=f_{0}(1370)$} & \multicolumn{4}{|c|}{$m_{3}=f_{0}(1500)$} & \multicolumn{4}{|c|}{$m_{4}=f_{0}(1710)$} \\
\hline & $u+d$ & $s$ & $G$ & $G^{\prime}$ & $u+d$ & $s$ & $G$ & $G^{\prime}$ & $u+d$ & $s$ & $G$ & $G^{\prime}$ & $u+d$ & $s$ & $G$ & $G^{\prime}$ \\
\hline 0.67 & 0.86 & 0.00 & 0.14 & 0.01 & 0.13 & 0.04 & 0.83 & 0.04 & 0.01 & 0.01 & 0.00 & 0.98 & 0.01 & 0.96 & 0.03 & 0.01 \\
\hline 0.68 & 0.90 & 0.00 & 0.05 & 0.05 & 0.04 & 0.01 & 0.94 & 0.01 & 0.05 & 0.06 & 0.00 & 0.89 & 0.01 & 0.93 & 0.01 & 0.05 \\
\hline$\mu$ & \multicolumn{3}{|c|}{$R\left(m_{2} / m_{1}\right)$} & \multicolumn{3}{|c|}{$R\left(m_{3} / m_{1}\right)$} & \multicolumn{2}{|c|}{$R\left(m_{4} / m_{1}\right)$} & \multicolumn{3}{|c|}{ A } & $\nu$ & \multicolumn{3}{|c|}{$\nu^{\prime}$} & $\delta$ \\
\hline 0.67 & \multicolumn{3}{|c|}{4.17} & \multicolumn{3}{|c|}{4.21} & \multicolumn{2}{|r|}{0.11} & \multicolumn{3}{|c|}{0.08} & 0.40 & \multicolumn{3}{|c|}{0.13} & 0.47 \\
\hline 0.68 & \multicolumn{3}{|c|}{7.07} & \multicolumn{3}{|c|}{5.73} & \multicolumn{2}{|r|}{0.26} & \multicolumn{3}{|c|}{0.07} & 0.25 & \multicolumn{3}{|c|}{0.35} & 0.40 \\
\hline
\end{tabular}

${ }^{1} S_{0}$ and ${ }^{5} D_{0}$ chromoball states. Again this is consistent with Table II.

As for the $J / \psi$ radiative decay branching ratio, Table III shows that $R\left(f_{0}(1710) / f_{0}(1500)\right) \simeq 0.8$ when $\mu=0.60$, and Table IV gives around 0.05 when $\mu=680 \mathrm{MeV}$. Clearly, both are too small compared to (44), so that we cannot tell which is the isotriplet partner of the $0^{++}$ isosinglet state.

The contrast between Tables III and IV is unmistakable. This, of course, originates from the inputs (42) and (43). This analysis has both positive and negative sides. The positive side is that the result of the $4 \times 4$ mixing, in particular the physical contents of the mass eigenstates, is consistent with the $3 \times 3$ mixing analysis. However, the disappointing point is that the $4 \times 4$ analysis cannot tell whether the PDG view that $a_{0}(1450)$, not $a_{0}(980)$, is the isotriplet partner of the $0^{++}$isosinglet state.

So far we have excluded $f(500)$ in the mixing, because it could be viewed as a neuroball or monoball, not a chromoball. On the other hand, there is no reason why it cannot mix with quarkoniums and chromoballs. Actually, even when $f_{0}(500)$ becomes a neuroball it makes sense to include it in the mixing, because the neuroball could be viewed as a glueball. This must be clear from Figs. 6 and 7 . Moreover, even when it becomes the monoball, the vacuum fluctuation of the monopole condensation, there is no reason why it could not mix with quarkoniums and chromoballs. This justifies the $5 \times 5$ mixing.

For this reason we consider the following $5 \times 5$ mixing with two $q \bar{q}$ and three glueballs which has nine parameters,

$$
M^{2}=\left(\begin{array}{ccccc}
E+\frac{2}{3} \Delta & -\frac{\sqrt{2}}{3} \Delta & 0 & 0 & 0 \\
-\frac{\sqrt{2}}{3} \Delta & E+\frac{1}{3} \Delta+3 A & \nu_{0} & \nu_{1} & \nu_{2} \\
0 & \nu_{0} & G_{0} & 0 & 0 \\
0 & \nu_{1} & 0 & G_{1} & 0 \\
0 & \nu_{2} & 0 & 0 & G_{2}
\end{array}\right),
$$

Here, $G_{1}$ and $G_{2}$ are the ${ }^{1} S_{0}$ and ${ }^{5} D_{0}$ chromoball as before, but $G_{0}$ is supposed to be the monoball or the neuroball.
In this case, we can put all five physical states below $2 \mathrm{GeV}$, including $f_{0}(500)$, and adopt (42) or (43) as the input, and treat $\mu$ as a free parameter. However, we need to impose one more constraint to fix the mass matrix completely.

To do that, we may have to take into account the possibility that $G_{0}$ is not an ordinary chromoball. There are two possibilities. If $f_{0}(500)$ is the neuroball, it is natural to expect $\nu_{0}$ to be of the same order as $\nu_{1}$ and $\nu_{2}$. This must be clear from the mixing diagram Fig. 7. In this case, we could assume

$$
\nu_{0} \simeq \frac{\nu_{1}+\nu_{2}}{2}
$$

On the other hand, if $f_{0}(500)$ is the monoball, its coupling to ordinary chromoball and quarkonium states could be of second order. In this case, $\nu_{0}$ could be much smaller than $\nu_{1}$ and $\nu_{2}$. Here, we will use (47) as the constraint. Of course, we emphasize that there is no justification for this. We assume this just for simplicity to fix the mass matrix completely.

Now, with

$$
G_{0}=4 \mu_{0}^{2}, \quad G_{1}=G_{0}+\delta=4 \mu^{2}, \quad G_{2}=G_{0}+\delta^{\prime},
$$

we obtain Table V using (42), and Table VI using (43). However, diagonalizing the $5 \times 5$ matrix involves solving a sixth order polynomial, and it is not easy to find the solution with the input data. So we have changed the input masses a little bit, and used 550, 990, 1400, 1505, and $1722 \mathrm{MeVs}$ for $f_{0}(500), f_{0}(980), f_{0}(1370), f_{0}(1500)$, and $f_{0}(1710)$ to obtain Table V, and 550, 990, 1370, 1505, and 1800 for $f_{0}(500), f_{0}(980), f_{0}(1370), f_{0}(1500)$, and $f_{0}(1710)$ to obtain Table VI.

Notice that here we have expressed $G_{0}$ in terms of the neuron effective mass $\mu_{0}$ (assuming that $G_{0}$ is the neuroball) to compare it with the chromon mass $\mu$ fixed by $G_{1}$. In this $5 \times 5$ mixing, the glueballs play the dominant role, because only two of the physical states can be the $q \bar{q}$ states. 
TABLE V. The numerical analysis of the $5 \times 5$ mixing in the $0^{++}$channel, with all five mass eigenstates $\left(f_{0}(500), f_{0}(980), f_{0}(1370)\right.$, $f_{0}(1500)$, and $f_{0}(1710)$ ) as the input. Here, $a_{0}(1450)$ is identified as the isotriplet partner.

\begin{tabular}{|c|c|c|c|c|c|c|c|c|c|c|c|c|c|c|c|c|c|c|c|c|c|}
\hline \multirow[b]{2}{*}{$\underline{\mu_{0}}$} & \multirow[b]{2}{*}{$\mu$} & \multicolumn{5}{|c|}{$m_{1}=f_{0}(500)$} & \multicolumn{5}{|c|}{$m_{2}=f_{0}(980)$} & \multicolumn{5}{|c|}{$m_{3}=f_{0}(1370)$} & \multicolumn{5}{|c|}{$m_{4}=f_{0}(1500)$} \\
\hline & & $u+d$ & $s$ & $G_{0}$ & $G_{1}$ & $G_{2}$ & $u+d$ & $s$ & $G_{0}$ & $G_{1}$ & $G_{2}$ & $u+d$ & $s$ & $G_{0}$ & $G_{1}$ & $G_{2}$ & $u+d$ & $s$ & $G_{0}$ & $G_{1}$ & $G_{2}$ \\
\hline 028 & 0.50 & & 0.00 & 1.00 & 0.00 & م & & 0.00 & 0.00 & 1.00 & 0.00 & & & 0.00 & 0.00 & & & 0.00 & 0.00 & 0.00 & 0.87 \\
\hline & & & & 97 & .00 & & & & 0.0 & & & & & & & & & .01 & & 0.02 & 0.79 \\
\hline 0.32 & 0.53 & .03 & 0.02 & 0.92 & 0.02 & 0.00 & 0.04 & 0.04 & 0.05 & 0.87 & 0.00 & & 0.79 & 0.00 & 0.01 & 0. & & 0.01 & 0.01 & 0.04 & 0.71 \\
\hline 034 & 0.55 & & 0.04 & 0.86 & 0.04 & 0.00 & & 0.05 & 0.0 & 0.80 & 0.01 & & 0.79 & 0.00 & 0.02 & 0. & & 0.01 & 0.02 & 0.08 & 0.63 \\
\hline 0.36 & 0.56 & 0.08 & 0.05 & 0.80 & 0.07 & 0.00 & 0.06 & 0.05 & 0.15 & 0.73 & 0.01 & 0.18 & 0.78 & 0.00 & 0.02 & 0.01 & 0.31 & 0.01 & 0.02 & 0.11 & 0.55 \\
\hline
\end{tabular}

\begin{tabular}{|c|c|c|c|c|c|c|c|c|c|c|c|c|c|c|c|}
\hline \multirow[b]{2}{*}{$\underline{\mu_{0}}$} & \multirow[b]{2}{*}{$\mu$} & \multicolumn{5}{|c|}{$m_{5}=f_{0}(1710)$} & \multirow[b]{2}{*}{$R\left(m_{2} / m_{1}\right)$} & \multirow[b]{2}{*}{$R\left(m_{3} / m_{1}\right)$} & \multirow[b]{2}{*}{$R\left(m_{4} / m_{1}\right)$} & \multirow[b]{2}{*}{$R\left(m_{5} / m_{1}\right)$} & \multirow[b]{2}{*}{$\mathrm{A}$} & \multirow[b]{2}{*}{$\nu_{1}$} & \multirow[b]{2}{*}{$\nu_{2}$} & \multirow[b]{2}{*}{$\delta$} & \multirow[b]{2}{*}{$\delta^{\prime}$} \\
\hline & & $u+d$ & $s$ & $G_{0}$ & $G_{1}$ & $G_{2}$ & & & & & & & & & \\
\hline 0.28 & 0.50 & 0.69 & 0.19 & 0.00 & 0.00 & 0.12 & 0.80 & 0.01 & 0.43 & 0.05 & 0.26 & 0.10 & 0.23 & 0.67 & 2.03 \\
\hline 0.30 & 0.52 & 0.60 & 0.16 & 0.01 & 0.03 & 0.19 & 0.78 & 0.01 & 0.41 & 0.09 & 0.20 & 0.29 & 0.40 & 0.71 & 2.09 \\
\hline 0.32 & 0.53 & 0.52 & 0.14 & 0.02 & 0.06 & 0.27 & 0.77 & 0.02 & 0.40 & 0.13 & 0.14 & 0.54 & 0.34 & 0.73 & 2.03 \\
\hline 0.34 & 0.55 & 0.44 & 0.12 & 0.02 & 0.08 & 0.35 & 0.79 & 0.02 & 0.39 & 0.17 & 0.09 & 0.61 & 0.38 & 0.74 & 2.03 \\
\hline 0.36 & 0.56 & 0.38 & 0.10 & 0.02 & 0.07 & 0.42 & 0.81 & 0.03 & 0.39 & 0.22 & 0.04 & 0.67 & 0.40 & 0.73 & 2.02 \\
\hline
\end{tabular}

So here the issue becomes which of the five physical states are the $q \bar{q}$ states, not the glueball states.

Table V tells that $f_{0}(1370)$ is predominantly the $s \bar{s}$ state, and $f_{0}(1710)$ becomes the mixed state about half of which is the $u \bar{u}+d \bar{d}$ state (and $f_{0}(1500)$ becomes the mixed states about quarter of which is the $u \bar{u}+d \bar{d}$ state). This is consistent with Table I. Moreover, the table tells the followings. First, $f_{0}(500)$ is the mainly the lowest energy glueball which could be interpreted as either the neuroball or the monoball. Second, $f_{0}(980)$ and $f_{0}(1500)$ are mainly the ${ }^{1} S_{0}$ and ${ }^{5} D_{0}$ chromoball states, and a considerable part of $f_{0}(1710)$ is made of ${ }^{5} D_{0}$ chromoball.

In comparison, Table VI tells that $f_{0}(980)$ and $f_{0}(1710)$ predominantly the $u \bar{u}+d \bar{d}$ and $s \bar{s}$ states, respectively.
This is in line with Table II. Moreover, this table tells that $f_{0}(500)$ is mainly the lowest energy the neuroball (or the monoball), and $f_{0}(1370)$ and $f_{0}(1500)$ are mainly the ${ }^{1} S_{0}$ and ${ }^{5} D_{0}$ chromoball states.

In the literature, there have been diverse interpretations of the scalar mesons. One of the popular views is that $f_{0}(500)$ and $f_{0}(980)$ are the tetra-quark states [52-63], $f_{0}(1370)$ and $f_{0}(1500)$ are the mixed state [64-70], and $f_{0}(1710)$ is a scalar glueball [71-73]. Another view is that $f_{0}(1370), f_{0}(1710), a_{0}(1450)$, and $K_{0}^{*}(1430)$ are the members of the flavor nonet, $f_{0}(1710)$ being mainly the $s \bar{s}$ state $[74,75]$. In this view, $f_{0}(1500)$ can naturally be identified as predominantly the glueball state. And this seems to be endorsed by PDG [12].

TABLE VI. The numerical analysis of the $5 \times 5$ mixing in the $0^{++}$channel, with all five mass eigenstates $\left(f_{0}(500), f_{0}(980)\right.$, $f_{0}(1370), f_{0}(1500)$, and $\left.f_{0}(1710)\right)$ as the input. Here, $a_{0}(980)$ is identified as the isotriplet partner.

\begin{tabular}{|c|c|c|c|c|c|c|c|c|c|c|c|c|c|c|c|c|c|c|c|c|c|}
\hline \multirow[b]{2}{*}{$\mu_{0}$} & \multirow[b]{2}{*}{$\mu$} & \multicolumn{5}{|c|}{$m_{1}=f_{0}(500)$} & \multicolumn{5}{|c|}{$m_{2}=f_{0}(980)$} & \multicolumn{5}{|c|}{$m_{3}=f_{0}(1370)$} & \multicolumn{5}{|c|}{$m_{4}=f_{0}(1500)$} \\
\hline & & $u+d$ & $s$ & $G_{0}$ & $G_{1}$ & $G_{2}$ & $u+d$ & $s$ & $G_{0}$ & $G_{1}$ & $G_{2}$ & $u+d$ & $s$ & $G_{0}$ & $G_{1}$ & $G_{2}$ & $u+d$ & $s$ & $G_{0}$ & $G_{1}$ & $G_{2}$ \\
\hline 0.28 & 0.66 & 0.01 & 0.00 & 0.99 & 0.00 & 0.00 & 0.85 & 0.00 & 0.01 & 0.12 & 0.01 & 0.12 & 0.03 & 0.00 & 0.83 & 0.01 & 0.01 & 0.02 & 0.00 & 0.00 & 0.97 \\
\hline 0.30 & 0.69 & 0.07 & 0.00 & 0.92 & 0.00 & 0.00 & 0.85 & 0.00 & 0.07 & 0.00 & 0.08 & 0.00 & 0.00 & 0.00 & 1.00 & 0.00 & 0.08 & 0.09 & 0.00 & 0.00 & 0.83 \\
\hline 0.32 & 0.68 & 0.13 & 0.00 & 0.86 & 0.00 & 0.01 & 0.77 & 0.00 & 0.14 & 0.01 & 0.08 & 0.00 & 0.00 & 0.00 & 1.00 & 0.00 & 0.08 & 0.10 & 0.00 & 0.00 & 0.82 \\
\hline 0.34 & 0.68 & 0.20 & 0.01 & 0.78 & 0.00 & 0.01 & 0.69 & 0.00 & 0.21 & 0.02 & 0.08 & 0.02 & 0.00 & 0.00 & 0.97 & 0.01 & 0.09 & 0.11 & 0.00 & 0.00 & 0.81 \\
\hline 0.36 & 0.68 & 0.26 & 0.01 & 0.71 & 0.01 & 0.02 & 0.61 & 0.00 & 0.28 & 0.03 & 0.07 & 0.03 & 0.01 & 0.00 & 0.95 & 0.02 & 0.09 & 0.11 & 0.00 & 0.00 & 0.80 \\
\hline
\end{tabular}

\begin{tabular}{|c|c|c|c|c|c|c|c|c|c|c|c|c|c|c|c|}
\hline \multirow[b]{2}{*}{$\mu_{0}$} & \multirow[b]{2}{*}{$\mu$} & \multicolumn{5}{|c|}{$m_{5}=f_{0}(1710)$} & \multirow[b]{2}{*}{$R\left(m_{2} / m_{1}\right)$} & \multirow[b]{2}{*}{$R\left(m_{3} / m_{1}\right)$} & \multirow[b]{2}{*}{$R\left(m_{4} / m_{1}\right)$} & \multirow[b]{2}{*}{$R\left(m_{5} / m_{1}\right)$} & \multirow[b]{2}{*}{ A } & \multirow[b]{2}{*}{$\nu_{1}$} & \multirow[b]{2}{*}{$\nu_{2}$} & \multirow[b]{2}{*}{$\delta$} & \multirow[b]{2}{*}{$\delta^{\prime}$} \\
\hline & & $u+d$ & $s$ & $G_{0}$ & $G_{1}$ & $G_{2}$ & & & & & & & & & \\
\hline 0.28 & 0.66 & 0.01 & 0.95 & 0.00 & 0.03 & 0.01 & 0.12 & 0.49 & 0.48 & 0.01 & 0.08 & 0.39 & -0.18 & 1.48 & 1.95 \\
\hline 0.30 & 0.69 & 0.01 & 0.90 & 0.00 & 0.01 & 0.09 & 0.13 & 0.62 & 0.44 & 0.03 & 0.04 & 0.02 & -0.48 & 1.52 & 1.88 \\
\hline 0.32 & 0.68 & 0.01 & 0.89 & 0.00 & 0.00 & 0.09 & 0.21 & 0.66 & 0.46 & 0.04 & 0.03 & 0.50 & 0.10 & 1.46 & 1.83 \\
\hline 0.34 & 0.68 & 0.01 & 0.88 & 0.01 & 0.01 & 0.10 & 0.31 & 0.70 & 0.50 & 0.04 & 0.01 & 0.19 & 0.51 & 1.40 & 1.77 \\
\hline 0.36 & 0.68 & 0.01 & 0.87 & 0.01 & 0.01 & 0.10 & 0.42 & 0.76 & 0.54 & 0.05 & 0.00 & 0.25 & 0.53 & 1.34 & 1.71 \\
\hline
\end{tabular}


Our analysis does not entirely support this. If we identify $a_{0}(1450)$ as the isotriplet partner, $f_{0}(1370)$ and $f_{0}(1710)$ become the $q \bar{q}$ states. However, according to Table V, $f_{0}(1370)$ turns out to be predominantly the $s \bar{s}$ state. On the other hand, Table VI shows that $f_{0}(1710)$ becomes predominantly the $s \bar{s}$ state, if we identify $a_{0}(980)$ as the isotriplet partner. So, at this point it is premature to make a definite conclusion on which state, $a_{0}(980)$ or $a_{0}(1450)$, is the isotriplet partner of the $0^{++}$isosinglet $q \bar{q}$ state. We just remark that here our analysis does show that the possibility that $a_{0}(980)$ could be the isotriplet partner remains an option.

However, we like to emphasize two remarkable results of our mixing. First, both tables seem to be consistent with the view that $f_{0}(500)$ is the neuroball. Moreover, they suggest that the neuron mass $\mu_{0}$ is around $300 \mathrm{MeV}$, which is smaller than the chromon mass. This is interesting and reasonable. This should be compared with the popular view that $f_{0}(500)$ (and $f_{0}(980)$ ) are the tetra-quark states. As we have pointed out, in our quark and chromon model the tetra-quark states could be interpreted as the glueballs made of two neurons or chromons. This must be clear from Figs. 5 and 6. So this result is not inconsistent with the popular view that $f_{0}(500)$ is a tetra-quark state.

Second, both tables suggest that $f_{0}(1500)$ could be predominantly the chromoball state. This is also very interesting. On the other hand, in both tables the radiative decay ratio $R\left(f_{0}(1710) / f_{0}(1500)\right)$ turns out to be too small compared to (44). However, we notice that the relative radiative decay ratios, in general, are very sensitive to the inputs, so that this could be due to the ad hoc constraint (47).

To summarize, it is difficult to draw any conclusive result from the above numerical analysis. Perhaps one positive side of the above analysis is that $a_{0}(980)$ could still turn out to be the isotriplet partner of the flavor octet in this channel. Another point is the physical content of $f_{0}(500)$. It has been a big mystery in hadron spectroscopy, on which a huge amount of literature exists [76-79]. In this paper, we studied the possibility that it could be interpreted as a neuroball. Our result appears to be consistent with this view. However, it could also turn out to be the monoball, and we certainly need more analysis to make a definite conclusion on this.

\section{B. $2^{++}$channel}

In this channel, we have three physical states below $2 \mathrm{GeV}, f_{2}(1270), f_{2}^{\prime}(1525)$, and $f_{2}(1950)$. On the other hand, we have to keep in mind that there is the fourth state $f_{2}(2010)$ just above $2 \mathrm{GeV}$, which could be included in the mixing. Another point is that PDG lists five more unestablished states, $f_{2}(1430), f_{2}(1565), f_{2}(1640), f_{2}(1810)$, and $f_{2}(1910)$, some of which could turn out to be real states. In this paper, we will consider only the three and $f_{2}(2010)$ established states in the mixing analysis, but the fact that there are so many unestablished $2^{++}$states implies that we have to be careful to analyse the mixing in this channel.

In the preceding paper, we have studied the $3 \times 3$ mixing of one chromoball and two quarkoniums, using

$$
\begin{aligned}
E & =m^{2}\left(a_{2}\right), \quad a_{2}=a_{2}(1320), \\
\Delta & =2\left(m^{2}\left(K^{*}\right)-m^{2}\left(a_{2}\right)\right), \\
K^{*} & =K_{2}^{*}(1430),
\end{aligned}
$$

with two physical states $f_{2}(1270)$ and $f_{2}(1950)$ as the input, and predicted the mass of the third physical state varying the chromon mass $\mu$ as a free parameter [32].

The result suggests that, when the mass parameter $\mu$ is around $760 \mathrm{MeV}, f_{2}(1270)$ becomes a mixture of $u \bar{u}+d \bar{d}$ and chromoball, $f_{2}(1950)$ becomes a mixture of $u \bar{u}+d \bar{d}$, $s \bar{s}$ and the chromoball, but $f^{\prime}(1525)$ becomes predominantly the $s \bar{s}$ state.

On the other hand, when $\mu$ becomes around $860 \mathrm{MeV}$, $f_{2}(1270)$ becomes predominantly $u \bar{u}+d \bar{d}$ state, $f_{2}(1950)$ becomes predominantly the chromoball, and $f_{2}^{\prime}(1525)$ remains predominantly the $s \bar{s}$ state. This was in line with the PDG suggestion, which interprets $f_{2}(1270)$ and $f_{2}^{\prime}(1525)$ as the $q \bar{q}$ states [12].

However, now we have more experimental data on the $J / \psi$ radiative decay from PDG [12]

$$
\begin{aligned}
& J / \psi \rightarrow \gamma f_{2}(1270) \simeq(1.43 \pm 0.11) \times 10^{-3}, \\
& J / \psi \rightarrow \gamma f_{2}^{\prime}(1525) \simeq(4.5+0.7-0.4) \times 10^{-4}, \\
& J / \psi \rightarrow \gamma f_{2}(1950) \simeq(7.0 \pm 2.2) \times 10^{-4},
\end{aligned}
$$

which give us

$$
\begin{aligned}
& R\left(f_{2}(1525) / f_{2}(1270)\right) \simeq 0.36, \\
& R\left(f_{2}(1950) / f_{2}(1270)\right) \simeq 0.49, \\
& R\left(f_{2}(1950) / f_{2}^{\prime}(1525)\right) \simeq 1.56 .
\end{aligned}
$$

So we could test these experimental data in our analysis.

In this paper, we first do the $3 \times 3$ mixing with all three inputs, $f_{2}(1270), f_{2}^{\prime}(1525)$, and $f_{2}(1950)$, with (49). In this case, we can fix all five mixing parameters, including the chromon mass $\mu$, completely. To find the solution, however, we have to vary the masses a bit. Changing the masses of $f_{2}(1270), f_{2}^{\prime}(1525)$, and $f_{2}(1950)$ to 1275 , 1515, and $1944 \mathrm{MeVs}$, we obtain Table VII which suggests the chromon mass to be around $920 \mathrm{MeV}$.

One might worry that $\mu \simeq 920 \mathrm{MeV}$ of Table VII is a bit too large. However, remember that here the $2^{++}$chromoball is ${ }^{5} S_{2}$ state in which the spin of two chromons are parallel. And the spin-spin interaction could have made the chromon mass large. So the large chromon mass here actually could be interpreted to include the energy coming from the spin-spin interaction. 
TABLE VII. The numerical analysis of the $3 \times 3$ mixing in the $2^{++}$channel, with $f_{2}(1270), f_{2}^{\prime}(1525)$ and $f_{2}(1950)$ as the input.

\begin{tabular}{|c|c|c|c|c|c|c|c|c|c|c|c|c|c|}
\hline \multirow[b]{2}{*}{$\mu$} & \multicolumn{3}{|c|}{$m_{1}=f_{2}^{\prime}(1270)$} & \multicolumn{3}{|c|}{$m_{2}=f_{2}^{\prime}(1525)$} & \multicolumn{3}{|c|}{$m_{3}=f_{2}(1950)$} & \multirow[b]{2}{*}{$R\left(m_{2} / m_{1}\right)$} & \multirow[b]{2}{*}{$R\left(m_{3} / m_{1}\right)$} & \multirow[b]{2}{*}{$A$} & \multirow[b]{2}{*}{$\nu$} \\
\hline & $u+d$ & $s$ & $G$ & $u+d$ & $s$ & $G$ & $u+d$ & $s$ & $G$ & & & & \\
\hline 0.92 & 0.86 & 0.01 & 0.13 & 0.04 & 0.88 & 0.07 & 0.10 & 0.10 & 0.80 & 0.41 & 2.33 & 0.07 & 0.79 \\
\hline
\end{tabular}

The result tells that $f_{2}(1270)$ is predominantly the $u \bar{u}+d \bar{d}$ state, $f_{2}^{\prime}(1525)$ is predominantly the $s \bar{s}$ state, and $f_{2}(1950)$ is predominantly the chromoball state. This agrees well with the result of the preceding paper, and is consistent with the PDG view [12,32].

Notice that the table gives us $R\left(f_{2}^{\prime}(1525) / f_{2}(1270)\right) \simeq$ 0.41 which agrees well with the PDG value 0.36 , but $R\left(f_{2}(1950) / f_{2}(1270)\right)$ becomes 2.33 which is a little larger than the PDG value (50). However, we find that we could reduce this number by changing the mass of $f_{2}(1525)$ to around $1490 \mathrm{MeV}$. With this change of the input, the chromon mass is reduced to around $840 \mathrm{MeV}$ and $R\left(f_{2}(1950) / f_{2}(1270)\right)$ becomes around 0.57 .

Now, remember that here we have $f_{0}(2010)$ just above $2 \mathrm{GeV}$, and it would be unfair to exclude this in the mixing. So we consider the $4 \times 4$ mixing with the four mass eigenstates and (49) as the input, and obtain Table VIII. Here, again, we have changed the input masses a little, to $1275,1500,1944$, and $2100 \mathrm{MeVs}$, to find the solution.

Remarkably, the result in Table VIII is very similar to the Table VII. Although the numbers are different, the general feature is the same. Here, again, $f_{2}(1270)$ becomes predominantly the $u \bar{u}+d \bar{d}$ state, $f_{2}^{\prime}(1525)$ becomes predominantly the $s \bar{s}$ state, and $f_{2}(1950)$ becomes predominantly a chromoball state. The only new thing is that $f_{2}(2010)$ becomes the second chromoball state, so that we can interpret $f_{2}(1950)$ and $f_{2}(2010)$ to be predominantly the ${ }^{5} S_{2}$ and ${ }^{1} D_{2}$ chromoballs.
The main difference between the two tables is the $J / \psi$ relative radiative decay ratio. This is because the ratio is very sensitive to the chromoball contents of the physical states, so that a small change of the chromoball contents influence the ratio significantly. In Table VIII, the radiative decay ratios turn out to be larger then the PDG values (50). However, we find that the ratios could be reduced to PDG values by changing the mass of $f_{2}^{\prime}(1525)$ to around $1490 \mathrm{MeV}$.

We can do the $4 \times 4$ mixing with the three mass eigenstates below $2 \mathrm{GeV}$ and (49) as the input, and try to predict the fourth state. The result is shown in Table IX. However, here again, we have changed the mass eigenvalues to 1275,1500 , and $1944 \mathrm{MeVs}$ to obtain the solutions.

Remarkably, it predicts that the mass of the fourth state is around $2100 \mathrm{MeV}$, which we can identify to be $f_{2}(2010)$. With this identification Table IX becomes very similar to Table VIII, which confirms that $f_{2}(1270)$ is predominantly the $u \bar{u}+d \bar{d}$ state, $f_{2}^{\prime}(1525)$ is predominantly the $s \bar{s}$ state, but $f_{2}(1950)$ and $f_{0}(2010)$ are predominantly the ${ }^{5} S_{2}$ and ${ }^{1} D_{2}$ chromoballs.

So, all in all the mixing in the $2^{++}$channel seems to work fine, and the upshot of our mixing is that $f_{2}(1950)$ and $f_{0}(2010)$ are predominantly the chromoball states. On the other hand, it is good to remember that there are different suggestions in the literature. Clearly, there have been claims that $f_{2}(1270)$ and $f_{2}^{\prime}(1525)$ are the $q \bar{q}$ states as PDG suggests [80-82]. On the other hand, there have been

TABLE VIII. The numerical analysis of the $4 \times 4$ mixing in the $2^{++}$channel, with $f_{2}(1270), f_{2}^{\prime}(1525), f_{2}(1950)$, and $f_{2}(2010)$ as the input.

\begin{tabular}{|c|c|c|c|c|c|c|c|c|c|c|c|c|c|c|c|c|}
\hline \multirow[b]{2}{*}{$\mu$} & \multicolumn{4}{|c|}{$m_{1}=f_{2}(1270)$} & \multicolumn{4}{|c|}{$m_{2}=f_{2}^{\prime}(1525)$} & \multicolumn{4}{|c|}{$m_{3}=f_{2}(1950)$} & \multicolumn{4}{|c|}{$m_{4}=f_{2}(2010)$} \\
\hline & $u+d$ & $s$ & $G$ & $G^{\prime}$ & $u+d$ & $s$ & $G$ & $G^{\prime}$ & $u+d$ & $s$ & $G$ & $G^{\prime}$ & $u+d$ & $s$ & $G$ & $G^{\prime}$ \\
\hline 0.90 & 0.80 & 0.01 & 0.19 & 0.00 & 0.06 & 0.85 & 0.09 & 0.00 & 0.13 & 0.14 & 0.72 & 0.01 & 0.01 & 0.00 & 0.01 & 0.99 \\
\hline 0.91 & 0.79 & 0.01 & 0.18 & 0.02 & 0.06 & 0.85 & 0.08 & 0.01 & 0.09 & 0.09 & 0.67 & 0.15 & 0.05 & 0.05 & 0.07 & 0.83 \\
\hline 0.92 & 0.79 & 0.01 & 0.17 & 0.03 & 0.06 & 0.85 & 0.07 & 0.01 & 0.06 & 0.06 & 0.61 & 0.27 & 0.09 & 0.08 & 0.14 & 0.69 \\
\hline 0.93 & 0.79 & 0.01 & 0.16 & 0.04 & 0.06 & 0.86 & 0.07 & 0.01 & 0.03 & 0.03 & 0.54 & 0.39 & 0.12 & 0.10 & 0.22 & 0.55 \\
\hline 0.94 & 0.78 & 0.01 & 0.16 & 0.04 & 0.06 & 0.86 & 0.07 & 0.02 & 0.01 & 0.01 & 0.44 & 0.53 & 0.14 & 0.12 & 0.33 & 0.41 \\
\hline$\mu$ & \multicolumn{3}{|c|}{$R\left(m_{2} / m_{1}\right)$} & \multicolumn{3}{|c|}{$R\left(m_{3} / m_{1}\right)$} & \multicolumn{2}{|c|}{$R\left(m_{4} / m_{1}\right)$} & \multicolumn{3}{|c|}{ A } & $\nu$ & \multicolumn{3}{|c|}{$\nu^{\prime}$} & $\delta$ \\
\hline 0.90 & \multicolumn{3}{|c|}{1.49} & \multicolumn{3}{|c|}{1.43} & \multicolumn{2}{|r|}{0.37} & \multicolumn{3}{|c|}{0.11} & 0.89 & \multicolumn{3}{|c|}{0.14} & 1.16 \\
\hline 0.91 & \multicolumn{3}{|c|}{1.62} & \multicolumn{3}{|c|}{1.26} & \multicolumn{2}{|r|}{0.34} & \multicolumn{3}{|c|}{0.14} & 0.91 & \multicolumn{3}{|c|}{0.44} & 0.94 \\
\hline 0.92 & \multicolumn{3}{|c|}{1.71} & \multicolumn{3}{|c|}{1.13} & \multicolumn{2}{|r|}{0.33} & \multicolumn{3}{|c|}{0.15} & 0.93 & \multicolumn{3}{|c|}{0.56} & 0.75 \\
\hline 0.93 & \multicolumn{3}{|c|}{1.78} & \multicolumn{3}{|c|}{1.04} & \multicolumn{2}{|r|}{0.31} & \multicolumn{3}{|c|}{0.17} & 0.96 & \multicolumn{3}{|c|}{0.62} & 0.56 \\
\hline 0.94 & \multicolumn{3}{|c|}{1.82} & \multicolumn{3}{|c|}{0.98} & \multicolumn{2}{|r|}{0.31} & \multicolumn{3}{|c|}{0.18} & 0.99 & & 0.62 & & 0.39 \\
\hline
\end{tabular}


TABLE IX. The numerical analysis of the $4 \times 4$ mixing in the $2^{++}$channel, with states $f_{2}(1270), f_{2}^{\prime}(1525), f_{2}(1950)$ as the input. The fourth state could be interpreted as $f_{2}(2010)$.

\begin{tabular}{|c|c|c|c|c|c|c|c|c|c|c|c|c|c|c|c|c|c|}
\hline \multirow[b]{2}{*}{$\mu$} & \multirow[b]{2}{*}{$m_{4}$} & \multicolumn{4}{|c|}{$m_{1}=f_{2}(1270)$} & \multicolumn{4}{|c|}{$m_{2}=f_{2}^{\prime}(1525)$} & \multicolumn{4}{|c|}{$m_{3}=f_{2}(1950)$} & \multicolumn{4}{|c|}{$m_{4}$} \\
\hline & & $u+d$ & $s$ & $G$ & $G^{\prime}$ & $u+d$ & $s$ & $G$ & $G^{\prime}$ & $u+d$ & $s$ & $G$ & $G^{\prime}$ & $u+d$ & $s$ & $G$ & $G^{\prime}$ \\
\hline 0.90 & 2.86 & 0.80 & 0.01 & 0.19 & 0.00 & 0.06 & 0.85 & 0.09 & 0.00 & 0.14 & 0.14 & 0.72 & 0.00 & 0.00 & 0.00 & 0.00 & 0.99 \\
\hline 0.91 & 2.11 & 0.79 & 0.01 & 0.18 & 0.02 & 0.06 & 0.85 & 0.08 & 0.01 & 0.09 & 0.09 & 0.68 & 0.14 & 0.05 & 0.05 & 0.06 & 0.84 \\
\hline 0.92 & 2.07 & 0.79 & 0.01 & 0.18 & 0.02 & 0.06 & 0.85 & 0.08 & 0.01 & 0.05 & 0.05 & 0.54 & 0.36 & 0.10 & 0.09 & 0.21 & 0.61 \\
\hline 0.93 & 2.07 & 0.79 & 0.01 & 0.18 & 0.03 & 0.06 & 0.86 & 0.07 & 0.01 & 0.02 & 0.02 & 0.42 & 0.54 & 0.13 & 0.11 & 0.33 & 0.43 \\
\hline 0.94 & 2.08 & 0.78 & 0.01 & 0.17 & 0.03 & 0.06 & 0.86 & 0.07 & 0.01 & 0.01 & 0.01 & 0.33 & 0.65 & 0.14 & 0.12 & 0.43 & 0.21 \\
\hline$\mu$ & & $m_{4}$ & \multicolumn{3}{|c|}{$R\left(m_{2} / m_{1}\right)$} & \multicolumn{4}{|c|}{$R\left(m_{3} / m_{1}\right)$} & \multicolumn{2}{|c|}{$R\left(m_{4} / m_{1}\right)$} & & A & \multicolumn{3}{|c|}{$\nu$} & $\epsilon$ \\
\hline 0.90 & & 2.86 & \multicolumn{3}{|c|}{1.48} & \multicolumn{4}{|c|}{0.03} & \multicolumn{2}{|c|}{0.37} & & 0.12 & \multicolumn{3}{|c|}{0.89} & 4.89 \\
\hline 0.91 & & 2.11 & \multicolumn{3}{|c|}{1.61} & \multicolumn{4}{|c|}{1.23} & \multicolumn{2}{|c|}{0.34} & & 0.14 & \multicolumn{3}{|c|}{0.91} & 0.98 \\
\hline 0.92 & & 2.07 & \multicolumn{3}{|c|}{1.75} & \multicolumn{4}{|c|}{1.20} & \multicolumn{2}{|c|}{0.33} & & 0.15 & \multicolumn{3}{|c|}{0.95} & 0.63 \\
\hline 0.93 & & 2.07 & \multicolumn{3}{|c|}{1.82} & \multicolumn{4}{|c|}{1.10} & \multicolumn{2}{|c|}{0.32} & & 0.16 & \multicolumn{3}{|c|}{0.98} & 0.46 \\
\hline 0.94 & & 2.08 & \multicolumn{3}{|c|}{1.85} & \multicolumn{4}{|c|}{1.01} & \multicolumn{2}{|c|}{0.31} & & 0.17 & \multicolumn{3}{|c|}{1.02} & 0.33 \\
\hline
\end{tabular}

assertions that they can be viewed as molecular states [83-86]. So we need more time to understand the physical contents of the $2^{++}$states clearly.

However, what really makes the mixing analysis complicated is the fact that experimentally we have five unestablished states here, $f_{2}(1430), f_{2}(1565), f_{2}(1640)$, $f_{2}(1810)$, and $f_{2}(1910)$ [12]. Some of them could turn out to be real states and make the mixing unreliable. So we need more experimental clarification on the unestablished states. Even if all remain unestablished, we have to explain why there are so many unestablished states in this channel.

\section{C. $0^{-+}$channel}

This channel has attracted special attention because of the octet-singlet mixing, the U(1) problem, PCAC etc. In this channel, we have five established states below $2 \mathrm{GeV}$, $\eta(548), \eta^{\prime}(958), \eta(1295), \eta(1405)$, and $\eta(1475)$, and one unestablished state $\eta(1760)$.

In the preceding paper, we discussed the $4 \times 4$ mixing of two chromoball states and two $q \bar{q}$ states, using

$$
\begin{aligned}
& E=m^{2}(\pi), \quad \pi=\pi(140), \\
& \Delta=2\left(m^{2}(K)-m^{2}(\pi)\right), \quad K=K(498),
\end{aligned}
$$

with $\eta^{\prime}(958), \eta(1405)$, and $\eta(1760)$ as the input.

The result showed that the mass of the fourth physical state becomes around $550 \mathrm{MeV}$, which could be interpreted to be $\eta(548)$. In this case, $\eta(548)$ turns out to be a mixture of $u \bar{u}+d \bar{d}$ and $s \bar{s}$, while $\eta^{\prime}(958)$ becomes predominantly a gg chromoball [32].

This is not satisfactory and not in line with PDG, which interprets $\eta^{\prime}(958)$ as predominantly a $q \bar{q}$ state. There are other problems. For example, the physical contents of $\eta(1405)$ and $\eta(1760)$ depended very much on the chromon mass.
Moreover, the $J / \psi$ radiative decay ratios in this table do not agree well with PDG values. Indeed, experimentally PDG has new data

$$
\begin{aligned}
& J / \psi \rightarrow \gamma \eta(548) \simeq(1.104 \pm 0.034) \times 10^{-3}, \\
& J / \psi \rightarrow \gamma \eta^{\prime}(958) \simeq(5.15 \pm 0.16) \times 10^{-3}, \\
& J / \psi \rightarrow \gamma \eta(1405 / 1475) \simeq 4.9 \times 10^{-3},
\end{aligned}
$$

which reveals

$$
\begin{aligned}
R\left(\eta^{\prime}(958) / \eta(548)\right) & \simeq 4.66, \\
R(\eta(1405 / 1475) / \eta(548)) & \simeq 4.44 .
\end{aligned}
$$

So, we need to explain this.

However, most critical defect of the $4 \times 4$ mixing is that it cannot explain all five physical states. This is the critical shortcoming of the $4 \times 4$ mixing. For this reason we discuss the $5 \times 5$ mixing in the following which could explain all five established states.

Consider the following mixing matrix,

$$
M^{2}=\left(\begin{array}{ccccc}
E+\frac{2}{3} \Delta & -\frac{\sqrt{2}}{3} \Delta & 0 & 0 & 0 \\
-\frac{\sqrt{2}}{3} \Delta & E+\frac{1}{3} \Delta+3 A & \nu_{1} & \nu_{2} & \nu_{3} \\
0 & \nu_{1} & G_{1} & 0 & 0 \\
0 & \nu_{2} & 0 & G_{2} & 0 \\
0 & \nu_{3} & 0 & 0 & G_{3}
\end{array}\right),
$$

which describes the mixing of three chromoball states with two quarkoniums below $2 \mathrm{GeV}$. This has nine parameters. Now, normally we could choose seven inputs, (51) and five mass eigenvalue, and treat $\mu$ as a free parameter. In this 
case, we need one more constraint, and might impose the following constraint

$$
\nu_{1}=\nu, \quad \nu_{2}=\frac{\nu_{1}+\nu_{3}}{2}, \quad \nu_{3}=\nu^{\prime},
$$

just for simplicity.

However, here we choose a slightly different input. We choose four mass eigenstates, $\eta^{\prime}(958), \eta(1295), \eta(1405)$, $\eta(1475)$, and $R\left(\eta(1475) / \eta^{\prime}(958)\right)=0.95$ of (53) in stead of $\eta(548)$. With this we could predict the mass of the fifth physical state. The reason is that, as we have pointed out the mathematical equations which we need to solve to diagonalize the mass matrix are very rigid, so that we could not find the solution when we use the five mass eigenstates as the inputs.

Assuming that $G_{3}$ is the $g g g$ chromoball, we may let

$$
G_{1}=4 \mu^{2}, \quad G_{2}=4 \mu^{2}+\delta, \quad G_{3}=9 \mu^{2}+\delta^{\prime} .
$$

Actually this is also artificial, because here $G_{1}, G_{2}, G_{3}$ are supposed to be the mass eigenstates of three chromoballs. Nevertheless, we adopt (56) here, because this could provide some insight on the chromon mass and their binding.

With (55) and (56) we obtain Table X. The result shows that the mass of the fifth physical state is around $520 \mathrm{MeV}$, which could be identified as $\eta(548)$. In this case $\eta(548)$ turns out to be a mixture of $u \bar{u}+d \bar{d}$ and $s \bar{s}$, while $\eta^{\prime}(958)$ becomes largely a mixture of $s \bar{s}$ and a $g g$ chromoball, with less than $20 \%$ contamination of $u \bar{u}+d \bar{d}$. And $\eta(1295)$ is made of more than $50 \% g g$ chromoball and less than $20 \%$ $u \bar{u}+d \bar{d}$ and $s \bar{s}$ each.

However, remarkably, the table shows that $\eta(1405)$ and $\eta(1475)$ are mainly the chromoball states. Moreover, the $J / \psi$ radiative decay ratios $R\left(\eta(1405) / \eta^{\prime}(958)\right)$ is perfect, although $R\left(\eta^{\prime}(958) / \eta(548)\right)$ looks a bit larger. This looks interesting and reasonable, considering the fact that we have imposed the ad hoc constraints (55) and (56).

Notice that $\delta^{\prime}$ turns out to be negative, which shows that the mass of the three chromon bound state is smaller than the sum of the chromon masses. This might be understood to imply that the three chromon binding is quite strong. On the other hand, this could also be an artefact of (56). For instance we could introduce a new chromon mass $\mu_{0}$ with $G_{3}=3 \mu_{0}^{2}$ as shown in the table, and avoid the negative binding energy.

In the literature, of course, we have different views. The popular view that PDG endorses is that $\eta(548)$ and $\eta^{\prime}(958)$ are predominantly the $u \bar{u}+d \bar{d}$ and $s \bar{s}$ states, and that $\eta(1295)$ and $\eta(1475)$ are the first radial excitations of $\eta(548)$ and $\eta^{\prime}$ (958) [87-89]. However, it is widely agreed that $\eta(1405)$ is indeed a pseudo-scalar glueball [90-95]. This is endorsed by PDG and by our analysis, although there exists a lattice result which might contradict with this view [11].

Our result implies that the spectrum of the light pseudoscalar mesons could be understood within the context of the quarkonium-chromoball mixing. Nevertheless, the idea that $\eta(1295)$ and $\eta(1475)$ could be the radial excitations of $\eta(548)$ and $\eta^{\prime}(958)$ should be taken seriously [87-89].

To see how this popular view fares in our quark and chromon model, we consider the $3 \times 3$ mixing with only three physical states, $\eta(548), \eta^{\prime}(958)$, and $\eta(1405)$, excluding the supposedly radially excited states $\eta(1295)$ and $\eta(1475)$. Normally in the $3 \times 3$ mixing we could use the three masses and (51) as the input to diagonalize the mass matrix, but in this case we could not find the solution. So we choose only two mass eigenvalues, $\eta^{\prime}(958)$ and $\eta(1405)$, and vary the mass of $\eta(548)$. With this we obtain Table XI.

Interestingly, when the mass of $\eta(548)$ becomes $510 \mathrm{MeV}$, the radiative decay ratio $R(\eta(1405) / \eta(958)) \simeq$ 1.2 becomes close to the experimental value 0.95 . In this

TABLE X. The numerical analysis of the $5 \times 5$ mixing in the $0^{-+}$channel. Here, we have used $\eta^{\prime}(958), \eta(1275), \eta(1405), \eta(1475)$,

\begin{tabular}{|c|c|c|c|c|c|c|c|c|c|c|c|c|c|c|c|c|c|c|c|c|c|}
\hline \multirow[b]{2}{*}{$\mu$} & \multirow[b]{2}{*}{$m_{5}$} & \multicolumn{5}{|c|}{$m_{1}=\eta^{\prime}(958)$} & \multicolumn{5}{|c|}{$m_{2}=\eta(1295)$} & \multicolumn{5}{|c|}{$m_{3}=\eta(1405)$} & \multicolumn{5}{|c|}{$m_{4}=\eta(1475)$} \\
\hline & & $u+d$ & $s$ & $G_{1}$ & $G_{2}$ & $G_{3}$ & $u+d$ & $s$ & $G_{1}$ & $G_{2}$ & $G_{3}$ & $u+d$ & $s$ & $G_{1}$ & $G_{2}$ & $G_{3}$ & $u+d$ & $s$ & $G_{1}$ & $G_{2}$ & $G_{3}$ \\
\hline 0.58 & 0.52 & 0.18 & 0.37 & 0.43 & 0.01 & 0.01 & 0.19 & 0.18 & 0.53 & 0.06 & 0.05 & 0.02 & 0.01 & 0.01 & 0.92 & 0.04 & 0.04 & 0.03 & 0.02 & 0.02 & 0.90 \\
\hline 0.58 & 0.52 & 0.18 & 0.36 & 0.44 & 0.01 & 0.00 & 0.17 & 0.16 & 0.50 & 0.15 & 0.02 & 0.05 & 0.04 & 0.04 & 0.82 & 0.05 & 0.02 & 0.02 & 0.01 & 0.02 & 0.93 \\
\hline 0.58 & 0.51 & 0.18 & 0.37 & 0.43 & 0.00 & 0.01 & 0.19 & 0.18 & 0.54 & 0.00 & 0.08 & 0.00 & 0.00 & 0.00 & 1.00 & 0.00 & 0.04 & 0.03 & 0.02 & 0.00 & 0.91 \\
\hline 0.58 & 0.52 & 0.17 & 0.35 & 0.46 & 0.02 & 0.00 & 0.14 & 0.13 & 0.44 & 0.30 & 0.00 & 0.12 & 0.10 & 0.10 & 0.68 & 0.00 & 0.00 & 0.00 & 0.00 & 0.00 & 1.00 \\
\hline
\end{tabular}
and $R\left(\eta(1475) / \eta^{\prime}(958)\right)=0.95$ as the input. The fifth state could be interpreted as $\eta(548)$.

\begin{tabular}{|c|c|c|c|c|c|c|c|c|c|c|c|c|c|c|c|c|}
\hline \multirow[b]{2}{*}{$\mu$} & \multirow[b]{2}{*}{$m_{5}$} & \multicolumn{5}{|c|}{$m_{5}$} & \multirow[b]{2}{*}{$R\left(m_{2} / m_{1}\right)$} & \multirow[b]{2}{*}{$R\left(m_{3} / m_{1}\right)$} & \multirow[b]{2}{*}{$R\left(m_{4} / m_{1}\right)$} & \multirow[b]{2}{*}{$R\left(m_{5} / m_{1}\right)$} & \multirow[b]{2}{*}{ A } & \multirow[b]{2}{*}{$\nu$} & \multirow[b]{2}{*}{$\nu^{\prime}$} & \multirow[b]{2}{*}{$\delta$} & \multirow[b]{2}{*}{$\delta^{\prime}$} & \multirow[b]{2}{*}{$\mu_{0}$} \\
\hline & & $u+d$ & $s$ & $G_{1}$ & $G_{2}$ & $G_{3}$ & & & & & & & & & & \\
\hline 0.58 & 0.52 & 0.58 & 0.41 & 0.01 & 0.00 & 0.00 & 0.90 & 1.12 & 0.95 & 0.03 & 0.36 & 0.40 & -0.17 & 0.62 & -0.89 & 0.487 \\
\hline 0.58 & 0.52 & 0.58 & 0.41 & 0.01 & 0.00 & 0.00 & 0.93 & 1.02 & 0.95 & 0.03 & 0.37 & 0.41 & -0.17 & 0.58 & -0.87 & 0.489 \\
\hline 0.58 & 0.51 & 0.58 & 0.41 & 0.01 & 0.00 & 0.00 & 0.89 & 1.17 & 0.95 & 0.03 & 0.36 & 0.40 & 0.01 & 0.63 & -0.91 & 0.485 \\
\hline 0.58 & 0.52 & 0.57 & 0.42 & 0.01 & 0.01 & 0.00 & 0.97 & 0.84 & 0.95 & 0.03 & 0.38 & 0.43 & -0.01 & 0.52 & -0.85 & 0.492 \\
\hline
\end{tabular}


TABLE XI. The numerical analysis of the $3 \times 3$ mixing in the $0^{-+}$channel. Here, we choose $\eta^{\prime}(958)$ and $\eta(1405)$ as the input and vary the mass of $\eta(548)$ to obtain the table. No solution can be found when $m(\eta(548))>541 \mathrm{MeV}$.

\begin{tabular}{|c|c|c|c|c|c|c|c|c|c|c|c|c|c|c|}
\hline \multirow[b]{2}{*}{$m(\eta(548))$} & \multirow[b]{2}{*}{$A$} & \multirow[b]{2}{*}{$\nu$} & \multirow[b]{2}{*}{$\mu$} & \multicolumn{3}{|c|}{$m_{1}=\eta(548)$} & \multicolumn{3}{|c|}{$m_{2}=\eta^{\prime}(958)$} & \multicolumn{3}{|c|}{$m_{3}=\eta(1405)$} & \multirow[b]{2}{*}{$R\left(m_{3} / m_{2}\right)$} & \multirow[b]{2}{*}{$R\left(m_{1} / m_{2}\right)$} \\
\hline & & & & $u+d$ & $s$ & $G$ & $u+d$ & $s$ & $G$ & $u+d$ & $s$ & $G$ & & \\
\hline 510 & 0.34 & 0.51 & 0.64 & 0.60 & 0.39 & 0.01 & 0.23 & 0.47 & 0.30 & 0.17 & 0.14 & 0.69 & 1.2 & 0.05 \\
\hline 520 & 0.41 & 0.55 & 0.60 & 0.56 & 0.43 & 0.01 & 0.16 & 0.33 & 0.51 & 0.28 & 0.24 & 0.48 & 0.49 & 0.03 \\
\hline 530 & 0.50 & 0.48 & 0.55 & 0.51 & 0.48 & 0.01 & 0.09 & 0.19 & 0.72 & 0.40 & 0.34 & 0.27 & 0.19 & 0.02 \\
\hline 540 & 0.58 & 0.22 & 0.49 & 0.47 & 0.53 & 0.00 & 0.01 & 0.03 & 0.95 & 0.52 & 0.43 & 0.04 & 0.02 & 0.003 \\
\hline
\end{tabular}

case, $\eta(548)$ becomes $60 \% u \bar{u}+d \bar{d}$ and $39 \% s \bar{s}$, but $\eta^{\prime}(958)$ becomes a mixture of $47 \% s \bar{s}$ and $30 \% \mathrm{gg}$. And $\eta(1405)$ becomes predominantly (69\%) a chromoball.

To understand the physical meaning of Table XI, we notice that the physical contents depend very much on the mass of $\eta(548)$. Moreover, as the mass approaches to the physical value $548 \mathrm{MeV}, \eta^{\prime}(958)$ becomes predominantly the glueball.

This is troublesome, and does not seem to support the PDG view (that $\eta(1295)$ and $\eta(1475)$ are the radial excitations of $\eta(548)$ and $\left.\eta^{\prime}(958)\right)$ at all. This implies that our result shown in Table $\mathrm{X}$ is at least as good as the PDG view, although this matter has to be studied more carefully.

In this section, we have extended and improved the numerical analysis of the quarkonium-chromoball mixing of the preceding paper in three channels $0^{++}, 2^{++}$, and $0^{-+}$ below $2 \mathrm{GeV}$, based on our quark and chromon model. Although the numerical results are still inconclusive, the results in this paper seem to work better.

Theoretically, it must be clear that the numerical mixing should be regarded as an approximation. Moreover, technically the equation we need to solve to diagonalize the mass matrix is very rigid and sensitive to the ad hoc constraints we have imposed.

With these shortcomings it is natural that our results are not perfect. Nevertheless, it is fair to say that the above mixing analysis does show that the quark and chromon model provides a conceptually simple way to understand the glueballs and their mixing with quarkoniums.

\section{DISCUSSIONS}

One of the main problems in hadron spectroscopy has been the identification of the glueballs. In this paper, we have made the numerical analysis of chromoball-quarkonium mixing to identify the glueballs, based on the quark and chromon model obtained by the Abelian decomposition [32]. Our mixing analysis is a rough approximation, but it does confirm that the glueballs (i.e., the chromoballs) play a fundamental role in the hadron spectroscopy, although in general (except for the oddballs) they exist as mixed states. In fact, the analysis tells that it is simply impossible to understand the meson spectroscopy without them.
Our analysis was able to pinpoint the glueball candidates below $2 \mathrm{GeV}$ successfully. Indeed, our results strongly indicate that $f_{0}(1500)$ in the $0^{++}$sector, $f_{2}(1950)$ in the $2^{++}$sector, and $\eta(1405)$ and $\eta(1475)$ in the $0^{-+}$sector become predominantly the glueballs. Some of them have been suggested to be the glueball states before, but some of them [e.g., $\eta(1475)]$ are our suggestion.

In our mixing analysis, we have also tried to settle other unresolved issues. First, in the $0^{++}$sector an important issue is what is the isotriplet $q \bar{q}$ partner of the isosinglet $q \bar{q}$. There are two contending views. The popular view endorsed by PDG is that $a_{0}(1450)$ is the isotriplet partner, but the opposite view suggests that $a_{0}(980)$ is the isotriplet parner [12,32]. The popular view appears intuitively strange because, if this is so, the strange flavor octet partner $K_{0}^{*}(1430)$ becomes lighter than $a_{0}(1450)$. So it is important to find out which view is correct, and why. We tried to resolve this issue in our mixing. Unfortunately, our analysis could not provide a conclusive answer on this, but it does imply that the opposite view is not completely excluded yet.

Another issue in this sector is the nature of $f_{0}(500)$, which has been a big mystery [52-58,76-79]. In the quark and chromon model, the chromons are supposed to be the constituent gluons, but logically we cannot exclude the possibility that the neurons could also form a loosely bound state. In this paper, we discussed this possibility. Our analysis suggests that $f_{0}(500)$ could be viewed a neuroball state, and this is independent of which state we choose to be the isotriplet partner. In our quark and chromon model, the neuroballs (if exist) should look very much like loosely bound states of two (or three) $q \bar{q}$ mesons or $g g$ chromoballs, and $f_{0}(500)$ nicely fits in this picture. Remarkably, this is consistent with the popular view advocated by many authors [52-58]. However, we emphasize that in detail two views are different. The one interprets $f_{0}(500)$ to be a glueball, but the other interprets it a molecular state.

A related issue is whether the monopole condensation in QCD could generate a $0^{++}$vacuum fluctuation mode or not $[21,22,32]$. Theoretically, this, of course, is a fundamental question. If the answer turns out to be in the affirmative, $f_{0}(500)$ would be a natural candidate of the vacuum fluctuation. This is a very interesting and attractive 
possibility which warrants further study. Here, we just emphasize that our analysis does not exclude this possibility.

The mixing in the $2^{++}$sector is rather straightforward because there are no controversial issues here. Here, we have three physical states below $2 \mathrm{GeV}$, and our result tells that $f_{2}(1275)$ and $f_{2}^{\prime}(1525)$ are the $u \bar{u}+d \bar{d}$ and $s \bar{s}$ states, respectively. This, of course, is in line with the PDG interpretation [12]. Moreover, our result tells that $f_{2}(1950)$ is predominantly the chromoball state, which agrees with our result in the preceding paper [32].

This sounds all very nice, but we have to swallow this with a grain of salt. The problem is that in this sector PDG shows that there are five unestablished states, and some of them could turn out to be real. And it is quite possible that this could give us a serious impact on the mixing analysis.

Finally, in the $0^{-+}$sector an important issue is whether $\eta(1295)$ and $\eta(1750)$ are the radial excitations of $\eta(548)$ and $\eta^{\prime}(958)$ or not [87-89]. Our mixing analysis provides a different picture. Our result tells that $\eta(1295)$ can be viewed as a mixed state made of more than 50\% $\mathrm{gg}$ chromoball and less than $20 \% u \bar{u}+d \bar{d}$ and $s \bar{s}$ each, and $\eta(1475)$ is mainly the $g g g$ chromoball state. This looks very interesting and reasonable, although we have yet to see which view is correct.

One of the problems in the mixing analysis is that the mathematical equations to diagonalize the mass matrix are very rigid and sensitive to the input data. This is troublesome because in reality we often do not have enough input data. This has forced us to impose ad hoc constraints like (47) and (55) which may have distorted the reality. However, this is a technical problem we could avoid when enough experimental data become available.

Independent of the details, however, we emphasize the conceptual simplicity and clarity of the quark and chromon model. As a natural generalization of the quark model it tells what are the glueballs made of and how they mix with quarkoniums without ambiguity. Most importantly, it provides us the general framework of the hadron spectroscopy in simple and clear terms.

Of course, there are other models of glueballs, in particular the constituent gluon model, which allow similar mixing analysis. In fact, superficially our mixing analysis is almost identical to the mixing in this model. As we have emphasized, however, the constituent model has the critical defect that it cannot tell exactly what are the constituent gluons. In comparison, our model tells what are the constituent gluons and what are the binding gluons which bind the constituent gluons. This is because our model is based on different logic, that QCD is made of two types of gluons which play different roles. No other model is based on this fact.

To amplify this point, consider the so-called "modelindependent" calculations of gluball spectrum, the QCD sum rule approach [6] and the lattice calculation $[10,11]$.
It has been assumed that these calculations are based on "the first principles" of QCD and thus regarded as model independent. However, we have to know what is the first principles of QCD before we know how to calculate the glueball spectrum. As we have emphasized, the Abelian decomposition reveals the hidden principles of QCD, which makes the old-fashioned first principles obsolete.

For instance, in the QCD sum rule approach people have been calculating the glueball mass with the conventional current operators made of two gauge field strengths, claiming that this is based on the model-independent first principles. However, the Abelian decomposition tells that actually there is a new and much simpler way to calculate the glueball mass, with the gauge-invariant current operators made of two chromons. And obviously the two methods will give us different results.

Exactly the same way, in the lattice calculation we can construct the glueballs implementing the Abelian decomposition on lattice or without implementing it. And again we get different results [27,28]. Clearly, in the conventional lattice glueball calculations, the ingredient of the glueballs is two or three gauge field strengths. In comparison, in our approach, the ingredient of the glueballs is two or three chromons, and obviously the chromons are totally different from the gauge field strengths. Consequently the two calculations should have different results.

These two examples clearly tells that we must understand the first principles of QCD first, before we actually make the "model-independent" calculations. As we have explained in the first part of the paper, the Abelian decomposition allows us to do that. And this is not a conjecture, but mathematically a well established fact in QCD [21-31,45-48]. This is the advantage of the quark and chromon model.

Before we close we emphasize that the quark and chromon model is not just a theoretical proposal. The underlying proposition of the model that there exist two types of gluons could be tested directly by experiment. We already have enough knowledge on how to differentiate the gluon jet from the quark jet experimentally [96-100]. Moreover, there has been a new proposal on how to separate different types of jets at LHC [101]. Using this knowledge, we could actually confirm the existence of two types of gluon jets experimentally. So we do have an unmistakable way to justify the quark and chromon model experimentally.

Obviously, our numerical results in this paper are not perfect and cannot explain everything. Nevertheless, they do demonstrate that the quark and chromon model is at least as good as any other model in the literature which describes the glueballs and their mixing with quarkoniums. Moreover, the numerical mixing analysis is not the only application of our model. The next application would be to implement the Abelian decomposition in the QCD sum rule and the lattice QCD calculations and obtain a better 
understanding of glueballs. The work in these directions is in progress.

\section{ACKNOWLEDGMENTS}

This work is supported in part by the National Natural Science Foundation of China (Grant No. 11575254 and No. 11805242),
China Scholarship Council, and Basic Science Research Program through the National Research Foundation of Korea funded by the Ministry of Education (Grants No. 2015-R1D1A1A0-1057578 and No. 2018R1D1A1B0-7045163), and by the Center for Quantum Spacetime at Sogang University.
[1] H. Fritzsch and P. Minkowski, Nuovo Cimento A 30, 393 (1975).

[2] P. G. O. Freund and Y. Nambu, Phys. Rev. Lett. 34, 1645 (1975).

[3] J. Kogut, D. Sinclair, and L. Susskind, Nucl. Phys. B114, 199 (1976).

[4] R. L. Jaffe and K. Johnson, Phys. Lett. 60B, 201 (1976).

[5] P. Roy and T. Walsh, Phys. Lett. 78B, 62 (1978).

[6] M. Shifman, A. Vainshtein, and V. Zakharov, Nucl. Phys. B147, 385 (1979).

[7] J. Coyne, P. Fishbane, and S. Meshkov, Phys. Lett. 91B, 259 (1980).

[8] M. Chanowitz, Phys. Rev. Lett. 46, 981 (1981).

[9] J. Cornwall and A. Soni, Phys. Lett. 120B, 431 (1983).

[10] G. S. Bali, K. Schilling, A. Hulsebos, A. C. Irving, C. Michael, and P. W. Stephenson (UKQCD Collaboration), Phys. Lett. B 309, 378 (1993).

[11] C. J. Morningstar and M. Peardon, Phys. Rev. D 60, 034509 (1999).

[12] K. Olive et al. (Particle Data Group), Chin. Phys. C 38, 090001 (2014).

[13] C. Amsler and N. Tornqvist, Phys. Rep. 389, 61 (2004).

[14] D. V. Bugg, Phys. Rep. 397, 257 (2004).

[15] E. Klempt and A. Zaitsev, Phys. Rep. 454, 1 (2007).

[16] V. Mathieu, N. Kochelev, and V. Vento, Int. J. Mod. Phys. E 18, 1 (2009).

[17] W. Ochs, J. Phys. G 40, 043001 (2013).

[18] A. Chodos, R. Jaffe, K. Jhonson, C. Thorn, and V. Weisskopf, Phys. Rev. D 9, 3471 (1974).

[19] J. Dudek et al., Eur. Phys. J. A 48, 187 (2012).

[20] D. Dutta et al. (PANDA Collaboration), Nucl. Phys. A862, 231 (2011).

[21] Y. M. Cho, Phys. Rev. D 21, 1080 (1980).

[22] Y. M. Cho, Phys. Rev. Lett. 46, 302 (1981).

[23] Y. M. Cho, Phys. Rev. D 23, 2415 (1981).

[24] Y. S. Duan and M. L. Ge, Sci. Sin. 9, 1072 (1979).

[25] S. Kato, K. Kondo, T. Murakami, A. Shibata, T. Shinohara, and S. Ito, Phys. Lett. B 632, 326 (2006).

[26] S. Ito, S. Kato, K. Kondo, T. Murakami, A. Shibata, and T. Shinohara, Phys. Lett. B 645, 67 (2007).

[27] N. Cundy, Y. M. Cho, W. Lee, and J. Leem, Phys. Lett. B 729, 192 (2014).

[28] N. Cundy, Y. M. Cho, W. Lee, and J. Leem, Nucl. Phys. B895, 64 (2015).
[29] W. S. Bae, Y. M. Cho, and S. W. Kimm, Phys. Rev. D 65 , 025005 (2001).

[30] Y. M. Cho, Franklin H. Cho, and J. H. Yoon, Phys. Rev. D 87, 085025 (2013).

[31] Y. M. Cho, Int. J. Mod. Phys. A 29, 1450013 (2014).

[32] Y. M. Cho, X. Y. Pham, P. Zhang, J.-J. Xie, and Li-Ping Zou, Phys. Rev. D 91, 114020 (2015). Notice that Table IV in this paper has a typological error which is corrected in Table I in the present paper.

[33] J. Schwinger, Phys. Rev. 82, 664 (1951).

[34] Y. M. Cho and D. G. Pak, Phys. Rev. Lett. 86, 1947 (2001); 91, 039101 (2003).

[35] W. S. Bae, Y. M. Cho, and D. G. Pak, Phys. Rev. D 64, 017303 (2001).

[36] D. Gross and F. Wilczek, Phys. Rev. Lett. 30, 1343 (1973).

[37] H. Politzer, Phys. Rev. Lett. 30, 1346 (1973).

[38] V. Schanbacher, Phys. Rev. D 26, 489 (1982).

[39] Y. M. Cho and D. G. Pak, Phys. Rev. D 65, 074027 (2002).

[40] Y. M. Cho, M. L. Walker, and D. G. Pak, J. High Energy Phys. 05 (2004) 073.

[41] G. 't Hooft, Nucl. Phys. B190, 455 (1981).

[42] Y. M. Cho, Phys. Rev. D 62, 074009 (2000).

[43] Y. M. Cho, Phys. Rev. Lett. 44, 1115 (1980).

[44] Our notation of the $\lambda$-matrices is the same as the Gellman's notation, except that $\lambda_{5}$ has the opposite signature.

[45] L. Faddeev and A. J. Niemi, Phys. Rev. Lett. 82, 1624 (1999).

[46] S. Shabanov, Phys. Lett. B 458, 322 (1999); 463, 263 (1999).

[47] H. Gies, Phys. Rev. D 63, 125023 (2001).

[48] R. Zucchini, Int. J. Geom. Methods Mod. Phys. 01, 813 (2004).

[49] K. Kondo, S. Kato, A. Shibata, and T. Shinohara, Phys. Rep. 579, 1 (2015).

[50] B. de Witt, Phys. Rev. 162, 1195 (1967); 162, 1239 (1967).

[51] See e.g., M. Peskin and D. Schroeder, An Introduction to Quantum Field Theory (Addison-Wesley, Reading, MA, 1995).

[52] H. Nagahiro and A. Hosaka, Phys. Rev. C 88, 055203 (2013).

[53] M. Napsuciale and S. Rodriguez, Phys. Rev. D 70, 094043 (2004).

[54] J. R. Pelaez and G. Rios, Phys. Rev. Lett. 97, 242002 (2006). 
[55] H.-X. Chen, A. Hosaka, and S.-L. Zhu, Phys. Lett. B 650, 369 (2007).

[56] F. Giacosa, Phys. Rev. D 75, 054007 (2007).

[57] J. T. Londergan, J. Nebreda, J. Pelaez, and A. Szczepaniak, Phys. Lett. B 729, 9 (2014).

[58] A. H. Fariborz, J. Schechter, S. Zarepour, and S.M. Zebarjad, Phys. Rev. D 90, 033009 (2014).

[59] M. Alford and R. Jaffe, Nucl. Phys. B578, 367 (2000).

[60] L. Maiani, F. Piccinini, A. D. Polosa, and V. Riquer, Phys. Rev. Lett. 93, 212002 (2004).

[61] H. J. Lee and N. I. Kochelev, Phys. Lett. B 642, 358 (2006).

[62] L. Maiani, A. D. Polosa, and V. Riquer, Phys. Lett. B 651, 129 (2007).

[63] H. J. Lee, N. I. Kochelev, and Y. Oh, Phys. Rev. D 87, 117901 (2013).

[64] F. E. Close and A. Kirk, Phys. Lett. B 483, 345 (2000).

[65] M. Ablikim et al. (BES Collaboration), Phys. Lett. B 607, 243 (2005).

[66] F. Giacosa, Th. Gutsche, V.E. Lyubovitskij, and A. Faessler, Phys. Rev. D 72, 094006 (2005).

[67] D. Parganlija, F. Giacosa, and D. H. Rischke, Phys. Rev. D 82, 054024 (2010).

[68] A. H. Fariborz, A. Azizi, and A. Asrar, Phys. Rev. D 91, 073013 (2015).

[69] A. Kirk, Phys. Lett. B 489, 29 (2000).

[70] A. H. Fariborz, A. Azizi, and A. Asrar, Phys. Rev. D 92, 113003 (2015).

[71] W. Lee and D. Weingarten, Phys. Rev. D 61, 014015 (1999).

[72] S. Janowski, F. Giacosa, and D. H. Rischke, Phys. Rev. D 90, 114005 (2014).

[73] F. Brunner and A. Rebhan, Phys. Rev. Lett. 115, 131601 (2015).

[74] C. Amsler and F. E. Close, Phys. Rev. D 53, 295 (1996).

[75] F. Close and A. Kirk, Eur. Phys. J. C 21, 531 (2001).

[76] L. S. Kisslinger and M. B. Johnson, Phys. Lett. B 523, 127 (2001).

[77] V. Vento, Phys. Rev. D 73, 054006 (2006).

[78] V. Vento, Phys. Rev. D 75, 055012 (2007).
[79] J. Pelaez, Phys. Rep. 658, 1 (2016).

[80] D. M. Li, H. Yu, and Q. X. Shen, J. Phys. G 27, 807 (2001).

[81] F. Giacosa, T. Gutsche, V. E. Lyubovitskij, and A. Faessler, Phys. Rev. D 72, 114021 (2005).

[82] Z.-C. Ye, X. Wang, X. Liu, and Q. Zhao, Phys. Rev. D 86, 054025 (2012).

[83] R. Molina, D. Nicmorus, and E. Oset, Phys. Rev. D 78, 114018 (2008).

[84] C. Garcia-Recio, L. S. Geng, J. Nieves, L. L. Salcedo, E. Wang, and J. J. Xie, Phys. Rev. D 87, 096006 (2013).

[85] J.-J. Xie and E. Oset, Eur. Phys. J. A 51, 111 (2015).

[86] J.-J. Xie, E. Oset, and L.-S. Geng, Phys. Rev. C 93, 025202 (2016).

[87] F. Close and A. Kirk, Phys. Lett. B 397, 333 (1997).

[88] T. Barnes, F. E. Close, P. R. Page, and E. S. Swanson, Phys. Rev. D 55, 4157 (1997).

[89] T. Gutsche, V. E. Lyubovitskij, and M. C. Tichy, Phys. Rev. D 79, 014036 (2009).

[90] F. E. Close, G. R. Farrar, and Z. Li, Phys. Rev. D 55, 5749 (1997).

[91] D. M. Li, H. Yu, and S. S. Fang, Eur. Phys. J. C 28, 335 (2003).

[92] L. Faddeev, A. J. Niemi, and U. Wiedner, Phys. Rev. D 70, 114033 (2004).

[93] M. Majewski, J. Phys. G 38, 035008 (2011).

[94] F. Wang, J. Chen, and J. Liu, Phys. Rev. D 92, 076004 (2015).

[95] H.-Y. Cheng, H.-N. Li, and K.-F. Liu, Phys. Rev. D 79, 014024 (2009).

[96] H. P. Nilles and K. H. Streng, Phys. Rev. D 23, 1944 (1981).

[97] Z. Fodor, Phys. Rev. D 41, 1726 (1990).

[98] J. Pumplin, Phys. Rev. D 44, 2025 (1991).

[99] J. Gallicchio and M. D. Schwartz, Phys. Rev. Lett. 107, 172001 (2011).

[100] P. Komiske, E. Metodiev, and M. Schwartz, J. High Energy Phys. 01 (2017) 110.

[101] E. M. Metodiev and J. Thaler, Phys. Rev. Lett. 120, 241602 (2018). 